\title{
Solving the Step-Tolled Bottleneck Model with General User Heterogeneity
}

\author{
Hongyu Chen ${ }^{1}$, Yang $\mathrm{Liu}^{2}$, and Yu (Marco) Nie ${ }^{* 1}$ \\ ${ }^{1}$ Department of Civil and Environmental Engineering \\ Northwestern University \\ 2145 Sheridan Road, Evanston, IL 60208 \\ ${ }^{2}$ Department of Industrial and Systems Engineering \\ National University of Singapore \\ Singapore
}

August 30, 2015

\begin{abstract}
Two new numerical methods, a semi-analytical method and an exact method, are proposed in this paper for solving the step-tolled user equilibrium problem for the bottleneck model with general user heterogeneity. The semi-analytical method transforms the step-tolled user equilibrium problem into a static, asymmetric traffic assignment problem with side constraints, which is formulated and solved as a variational inequality problem (VIP). Existence and uniqueness results are obtained by examining the properties of the VIP. The exact method, on the other hand, locates the equilibrium solution by enumerating all possible combinations of user departure orders. To improve the computational efficiency of the exact method, a simple heuristic is developed to reduce the number of solutions to be enumerated. Examples of up to 1000 classes are tested. The results confirm that ignoring user heterogeneity may lead to sub-optimal design of step tolls. In all experiments, the semi-analytical method is found to be more efficient than the heuristic version of the exact method by an order of magnitude, albeit they consistently produce nearly identical equilibrium solutions.
\end{abstract}

Keywords: step toll; general user heterogeneity; bottleneck model; semi-analytical method; variational inequality problem

\section{Introduction}

The classic morning commute analyses based on the bottleneck model (Vickrey, 1969; Hendrickson and Kocur, 1981; Smith, 1984; Daganzo, 1985; Cohen, 1987; Newell, 1987; Arnott et al., 1990,

*Corresponding author, E-mail: y-nie@northwestern.edu; Phone: 1-847-467-0502.

(c) 2015. This manuscript version is made available under the Elsevier user license http://www.elsevier.com/open-access/userlicense/1.0/ 
1993; Yang and Huang, 1997; Liu and Nie, 2011) views the rush-hour traffic pattern as a user equilibrium of competing commuters, who seek to minimize their own cost associated with both queuing and schedule delays by making departure time (and in some cases also route) choices. An important policy implication from these analyses is that the rush-hour congestion may be significantly reduced through congestion pricing. Vickrey (1969), for example, had shown that a time-varying toll could completely eliminate queuing delay, and thereby maximizing system efficiency. Despite its theoretical appeal, however, a continuously varying toll is rarely implemented in practice. Instead, a so-called step toll is often adopted in practice, which keeps toll rates constant in predefined discrete time windows (see e.g. Lindsey et al., 2012, for a recent review). Researchers have reported that travelers dislike time-varying tolls more than flat tolls (Higgins, 1994; Chu, 1999).

User heterogeneity has attracted much attention in the morning commute analysis because of its potential impacts on the user equilibrium solutions and welfare effects of demand management policies (Small, 1982; Cohen, 1987; Arnott et al., 1992, 1994; Lindsey, 2004; Small et al., 2005; van den Berg and Verhoef, 2011b; Liu and Nie, 2011; Hall, 2013; Liu et al., 2015). In our context, user heterogeneity typically consists of the desired arrival time $\left(t^{*}\right)$, the value of time $(\alpha)$ and the values of schedule punctuality ( $\beta$ for early arrival and $\gamma$ for late arrival). Previous studies often make assumptions about the relationship between $\alpha, \beta$ and $\gamma$ to simplify the analysis. These assumptions range from the most restrictive "proportional heterogeneity" (Vickrey, 1973; Xiao et al., 2011; Arnott and Kraus, 1995; van den Berg and Verhoef, 2011a; van den Berg, 2014) or a less restrictive structure of heterogeneity that allows $\alpha$ and $\beta$ to vary freely while assuming $\gamma$ is proportional to $\beta$ (Cohen, 1987; Newell, 1987; Arnott et al., 1988, 1994; van den Berg and Verhoef, 2011b; Liu and Nie, 2011; Hall, 2013). Yet, since welfare effects of congestion pricing could vary substantially with the imposed heterogeneity structure (van den Berg and Verhoef, 2011b), it is unclear how well these restricted systems would approximate the real world with general user heterogeneity. This remains an open question, for restricting the heterogeneity structure one way or another has so far been considered necessary to maintain tractability. Numerical methods proposed in this paper address exactly this question. ${ }^{1}$

Recently, Liu et al. (2015) proposed a semi-analytical method for solving the bottleneck model with general user heterogeneity. Their approach transforms the dynamic user equilibrium (DUE) problem of the bottleneck model into an equivalent static asymmetric traffic assignment problem, which is then formulated and solved as a variational inequality problem (VIP). The first part of the paper further develops their semi-analytical approach to tackle the step tolled problem with general user heterogeneity. Unlike Liu et al. (2015), we formulate the commute cost function in the unit of travel time instead of travel cost in order to handle the capacity constraint, which ensures the number of commuters served in each step toll window does not exceed the limit imposed by the bottleneck capacity. We prove that an equivalent asymmetric traffic assignment problem (TAP) also exists for the step-tolled DUE problem and give the corresponding VIP formulation. A specialized algorithm that includes two loops is developed to solve the VIP. The outer loop iteratively determines the penalty (interpreted as extra delay) associated with the capacity constraint, and the inner loop solves an unconstrained VIP with the extra delay

\footnotetext{
${ }^{1}$ This paper still assumes the same desired arrival time $t^{*}$ for all travelers. At the end of the paper, we briefly explain how this might be relaxed.
} 
determined from the outer loop.

While the semi-analytical approach is convenient for finding step-tolled user equilibrium solutions, it is not particularly amenable to designing optimal step toll scheme because of the difficulty of relating design parameters to the corresponding equilibrium solutions in closed form. In light of this limitation, a new numerical scheme, referred to as the exact method here, is developed in this paper to locate an exact solution by enumerating all possible combinations of the commuter classes' presence and departure orders within each arrival window. The underlying idea is that, once a combination is given, an equilibrium solution can be easily solved from a linear equation system, thanks to the affine property of travel cost function, and subsequently verified against the equilibrium conditions. Our focus here is twofold. The first has to do with conceptualizing the method and establishing its correctness. The second focus is concerned with the operationalization of the exact method, noting that it is impractical except for extremely small problems since the number of possible departure orders grow exponentially with the problem size (i.e., the number of users and the number of toll windows). In particular, a heuristic method is developed to guide the search direction for the commuter classes' presence and departure orders. Our preliminary tests suggest that the heuristic method consistently finds the same solution identified by either the exact method (when the exact method can be used), or the semi-analytical method. The proposed numerical methods are then used in numerical experiments to demonstrate their utility in analyzing step toll designs.

In what follows, Section 2 first introduces notations and presents the bottleneck model with general user heterogeneity and step toll. Sections 3 and 4 present, respectively, the semi-analytical method and the exact method. Results of numerical examples are reported in Section 5 and Section 6 concludes the paper with a summary of main findings and directions for future research.

\section{Step-tolled bottleneck model with general user heterogeneity}

Consider a fixed number of individuals $(N)$ who commute from home to work through a corridor during morning rush hour. If the incoming flow rate exceeds its capacity $s$, a bottleneck will form and a first-in-first-out (FIFO) queue develops. Accordingly, the en-route travel time of any individual who departs from the bottleneck at time $t$ (corresponding to the arrival time at the workplace) contains two parts: a waiting time $T(t)$ in the queue and a free flow travel time $T_{0}$. Because the free flow travel time $T_{0}$ will not affect the following analysis, we assume $T_{0}=0$. It is also assumed that all commuters prefer to pass the bottleneck and arrive at work at $t^{*}$. However, due to the restriction of the bottleneck, some must depart earlier and others later. Whenever an early or late arrival occurs, a schedule cost is incurred. At user equilibrium, each commuter chooses an arrival time $t$ at the workplace to minimize a general cost which includes travel delay $T(t)$ and schedule cost.

To model user heterogeneity, commuters are divided into $n$ classes, each including $N_{i}(i=$ $1, \ldots, n)$ commuters who have identical unit cost of travel time $\left(\alpha_{i}\right)$ and unit cost of schedule delay ( $\beta_{i}$ for early arrival and $\gamma_{i}$ for late arrival). To guarantee the existence of equilibrium, it is assumed that $\beta_{i}<\alpha_{i}, \forall i$ (Lindsey, 2004). To avoid potential degenerative solutions, we also assume $\beta_{i} / \alpha_{i} \neq \beta_{j} / \alpha_{j}$ and $\gamma_{i} / \alpha_{i} \neq \gamma_{j} / \alpha_{j}$ for any $i \neq j$. Let $m^{E}$ denote the number of toll steps for commuters arriving at workplace before $t^{*}$ and let $m^{L}$ denote the number of toll steps for 


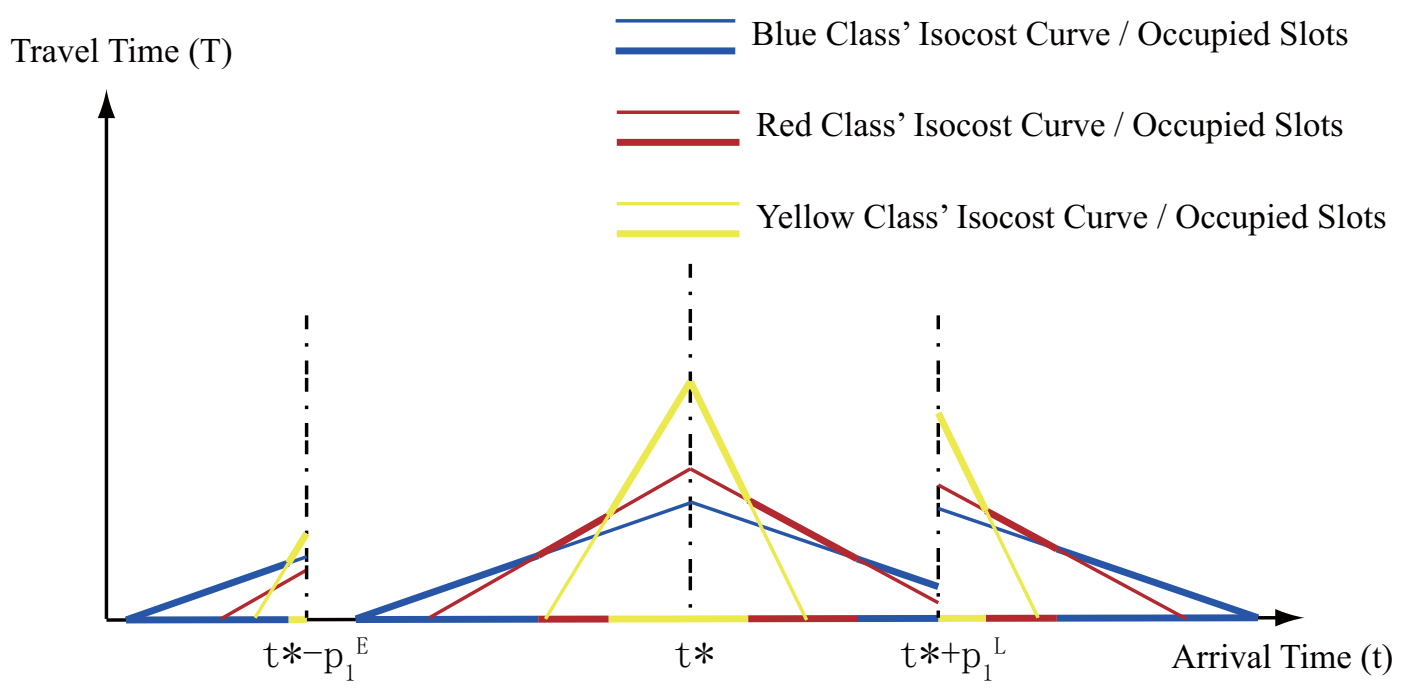

Figure 1: Illustration of isocurves at UE (Thick lines depict the upper envelop of the isocost curves)

commuters arriving at workplace after $t^{*}$. And let $\pi_{j}^{A}\left(j=1, \ldots, m^{A}, A=E, L\right)$ denote the uniform toll imposed within time windows $\left[t^{*}-p_{j}^{E}, t^{*}-p_{j+1}^{E}\right]$ or $\left[t^{*}+p_{j+1}^{L}, t^{*}+p_{j}^{L}\right]$, where $p_{j}^{A}$ is the time distance between $t^{*}$ and the start (end) time of $j^{\text {th }}$ step toll for early (late) arrival windows, and $p_{m^{A}+1}^{A}=0(A=E, L)$. A detailed description of the parameters and variables may be found in Appendix A.

A user equilibrium (UE) is attained if no commuter could reduce his/her commute cost by changing the departure time. The equilibrium solution may be illustrated and analyzed graphically using an isocost curve (e.g., Hendrickson and Kocur, 1981; Newell, 1987; Cohen, 1987; Arnott et al., 1994; Lindsey, 2004). Figure 1 shows the isocost curves in a three-class UE problem under a single-step toll scheme. Commuters may be seen as bidding a desired arrival time slot using their experienced queuing delay as the "currency". The isocost curve of each class actually represents the class's willingness to pay for each arrival time slot. At UE, an arrival time slot is always assigned to the class with highest willingness to pay. Hence, the isocost curve for each class of commuters changes in height so that each class obtains just enough time slots to cover their demand. Graphically, this means that commuters should always stay on the upper envelope of all the isocost curves at UE, whereas the corresponding arrival time slots in $x$-axis are "assigned" to them and the total length of these time slots equals $N_{i} / s$.

The isocost curve analysis corresponds to the definition of equilibrium, i.e., nobody can reduce his/her commute cost by changing the departure time. To explain it mathematically, let us use the example in Figure 1. The commute cost of class $i$ (expressed in the unit of travel time) can be formulated as a function of commuter's arrival time $t$ : 


$$
\mu_{i}(t)=\left\{\begin{array}{ccc}
T(t)+\frac{\beta_{i}}{\alpha_{i}}\left(t^{*}-t\right), & t<t^{*}-p_{1}^{E} & \text { untolled early arrival window } \\
T(t)+\frac{\beta_{i}}{\alpha_{i}}\left(t^{*}-t\right)+\frac{\pi_{1}^{E}}{\alpha_{i}}, & t^{*}-p_{1}^{E} \leq t<t^{*}-p_{2}^{E} & \text { first tolled early arrival window } \\
\ldots & \\
T(t)+\frac{\beta_{i}}{\alpha_{i}}\left(t^{*}-t\right)+\frac{\pi_{m}^{E}}{\alpha_{i}}, & t^{*}-p_{m^{E}}^{E} \leq t<t^{*} & \text { last tolled early arrival window } \\
T(t)+\frac{\gamma_{i}}{\alpha_{i}}\left(t-t^{*}\right)+\frac{\pi_{m}^{L}}{\alpha_{i}}, & t^{*} \leq t<t^{*}+p_{m^{L}}^{L} & \text { first tolled late arrival window } \\
\ldots & & \\
T(t)+\frac{\gamma_{i}}{\alpha_{i}}\left(t-t^{*}\right)+\frac{\pi_{1}^{L}}{\alpha_{i}}, & t^{*}+p_{2}^{L} \leq t<t^{*}+p_{1}^{L} & \text { last tolled late arrival window } \\
T(t)+\frac{\gamma_{i}}{\alpha_{i}}\left(t-t^{*}\right), & t \geq t^{*}+p_{1}^{L} & \text { untolled late arrival window }
\end{array}\right.
$$

The equilibrium definition implies $d \mu_{i}(t) / d t=0$. Then, it gives the slope of the isocost curve:

$$
\frac{\mathrm{d} T}{\mathrm{~d} t}=\left\{\begin{array}{ccc}
\frac{\beta_{i}}{\alpha_{i}}, & t<t^{*} \quad \text { early arrival } \\
\frac{-\gamma_{i}}{\alpha_{i}}, & t \geq t^{*} \quad \text { late arrival }
\end{array} .\right.
$$

It is well known that the primary complexity in step toll analysis comes from the discontinuity when the price of toll drops at the end of a toll window (e.g., time $t^{*}+p_{1}^{L}$ in Figure 1). To compensate the sudden drop in the toll charge, the first commuter in the lower-toll window must experience a higher non-toll cost than the last commuter in the higher-toll window would do at UE. This calls for additional assumptions about how commuters behave at the end of the higherpriced tolling window. Arnott et al. (1990) assume that a mass arrival (MA) occurs at the point of toll drop such that the above two critical consumers would have the same "expected cost". The braking-induced idle (BI) assumption of Lindsey et al. (2012) implies that the bottleneck will be operated below capacity at the point of discontinuity. Both assumptions lead to substantial changes to the isocost curves: the MA assumption changes the cost functions for the commuters arriving in the mass, whereas the BI assumption changes the capacity of the time window before each toll drop (hence the commuters assigned to these time windows have to raise their isocost curves up to obtain more time slots). Consequently, they require a configuration of isocost curves different from that depicted in Figure 1, which complicates the analysis.

In contrast, Laih $(1994,2004)$ shows that, in the case of homogenous users, a separate-waiting (SW) assumption avoids the complexity of deviating from the simple configuration of isocost curves shown in Figure 1. The SW assumption introduces a separate waiting queue to "hold" some commuters so that those who come after them but are willing to pay a higher toll can jump in front of them. This resolves the violation of first-in-first-out principle because there are two queues. In effect, the assumption allows the aforementioned two critical commuters to have different waiting time (even though they pass the bottleneck at the same time), without changing anyone's preference for time window or the bottleneck capacity. It can be shown (omitted here for brevity) that this nice property of the SW assumption still holds in the heterogeneous case.

We note that the above three behavioral assumptions give somewhat different UE solutions. Yet the results are expected to be relatively insensitive to them, especially if the behavioral parameters fall into realistic ranges (see e.g. Nie, 2013). Therefore, the SW assumption is adopted in what follows for its simplicity. 


\section{Semi-analytical method}

\subsection{Formulation}

We proceed in this section to show that the step-tolled user equilibrium problem can be transformed into an equivalent static traffic assignment problem, along the same line as in Liu (2013) and Liu et al. (2015).

We first divide the time, according to the discrete toll rates, into $\left(m^{E}+m^{L}+2\right)$ windows, corresponding to $m^{E}$ early-arrival tolled windows, $m^{L}$ late-arrival tolled windows and 2 untolled windows. By setting $\pi_{0}^{A}=0$ and $p_{0}^{A}=\infty$, the two untolled windows are treated as tolled windows with zero tolls. Therefore, each arrival window represents a time period with a uniform toll rate either before or after $t^{*}$.

According to Arnott et al. (1994), the departure order of class $i$ can be determined based on their time inflexibility $\left(\beta_{i} / \alpha_{i}\right.$ for early arrival or $\gamma_{i} / \alpha_{i}$ for late arrival). Namely, the higher the time inflexibility, the closer the class will travel to their desired arrival time $t^{*}$. Define $E(i)(L(i))$ as the class ID of the class whose $\frac{\beta}{\alpha}\left(\frac{\gamma}{\alpha}\right)$ value ranks at the $i^{\text {th }}$ place in all classes in ascending order. Without loss of generality, let:

$$
E(i)=i
$$

Hence, we have:

$$
\begin{aligned}
& \frac{\beta_{1}}{\alpha_{1}}<\frac{\beta_{2}}{\alpha_{2}}<\ldots<\frac{\beta_{n}}{\alpha_{n}} \\
& \frac{\gamma_{L(1)}}{\alpha_{L(1)}}<\frac{\gamma_{L(2)}}{\alpha_{L(2)}}<\ldots<\frac{\gamma_{L(n)}}{\alpha_{L(n)}} .
\end{aligned}
$$

In the semi-analytical method, isocost curves are used to impose the user equilibrium condition within each arrival window, i.e., the travel cost of each class remains identical given the constant slope derived in Equation (2). The equilibrium conditions across the arrival windows are completed by treating each arrival window as a route in the equivalent TAP, and thereby solving the equivalent TAP actually achieves the user equilibrium conditions across arrival windows.

We now formally state the equivalent traffic assignment problem as follows (see Liu (2013) for more details).

Equivalent static traffic assignment problem: Consider a network with $n$ origin-destination pairs, each from node $i$ to $i+1$ corresponding to a user class $i$ with a demand $N_{i}$. As shown in Figure 2, there are $\left(m^{E}+m^{L}+2\right)$ routes connecting each O-D pair, representing the $\left(m^{E}+m^{L}+2\right)$ arrival windows. Each commuter class $i$ is assigned to the $\left(m^{E}+m^{L}+2\right)$ arrival windows (i.e., $m^{E}+m^{L}+2$ routes between node $i$ and $i+1$ ) based on the Wardrop principle (Wardrop, 1952). Let $N_{i}^{A_{j}}\left(i=1,2 \ldots, n, j=0,1, \ldots, m^{A}, A=E, L\right)$ denote the flows of class $i$ in arrival window $A_{j}$.

An extra side constraint is needed to ensure that the number of commuters assigned to each arrival window does not exceed the bottleneck capacity. Note that the capacity of each arrival window $A_{j}$ is shared by the aggregated flows of all classes assigned to that window, that is, the total flows on the $A_{j}$-th route of all O-D pairs in Figure 2 is subject to that constraint. We denote $D^{A_{j}}(i)$ as the ratio rank ID of the class whose inflexibility ranks at the $i^{\text {th }}$ place among all classes 


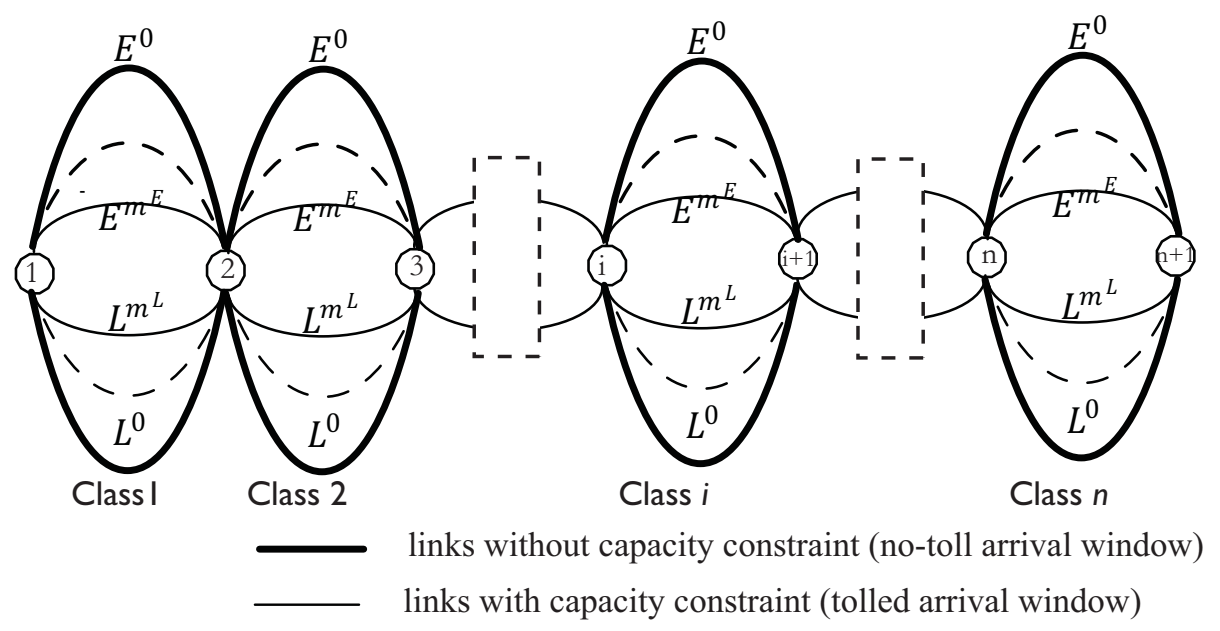

Figure 2: Network representation of the equivalent static traffic assignment problem

present in arrival window $A_{j}$. Hence, its true class ID should be $A\left(D^{A_{j}}(i)\right)(A=E, L)$. For early arrival windows, $E(i)=i$, hence the true class ID can be simplified as $D^{A_{j}}(i)$.

Figures 3(a) and 3(b) illustrate the cases with unsaturated and saturated capacity constraints, respectively. The maximum number of travelers that can pass the bottleneck within a tolled arrival window is determined by the window length $\left(p_{j+1}^{A}-p_{j}^{A}\right)$ and the bottleneck capacity $(s)$. If the capacity constraint of an arrival window $A_{j}$ is saturated, all commuters in the arrival window will be subject to an identical penalty $v_{j}^{A}$, which is the multiplier associated with the constraint and may be interpreted as an extra delay. Hence, we have the following complementarity conditions associated with the capacity constraint:

$$
\begin{array}{ll}
\left(\sum_{i=1}^{n} N_{i}^{A_{j}}-\left(p_{j+1}^{A}-p_{j}^{A}\right) s\right) v_{j}^{A}=0, & j=0,1, \ldots, m^{A}, A=E, L \\
\sum_{i=1}^{n} N_{i}^{A_{j}}-\left(p_{j+1}^{A}-p_{j}^{A}\right) s \leq 0, & j=0,1, \ldots, m^{A}, A=E, L \\
v_{j}^{A} \geq 0, & j=0,1, \ldots, m^{A}, A=E, L
\end{array}
$$

where $N_{i}^{A_{j}}$ is the number of commuters of class $i$ in the arrival window $A_{j}$.

In each time window $A_{j}$ and for the commuter of class $i$ furthest from the center, its travel 


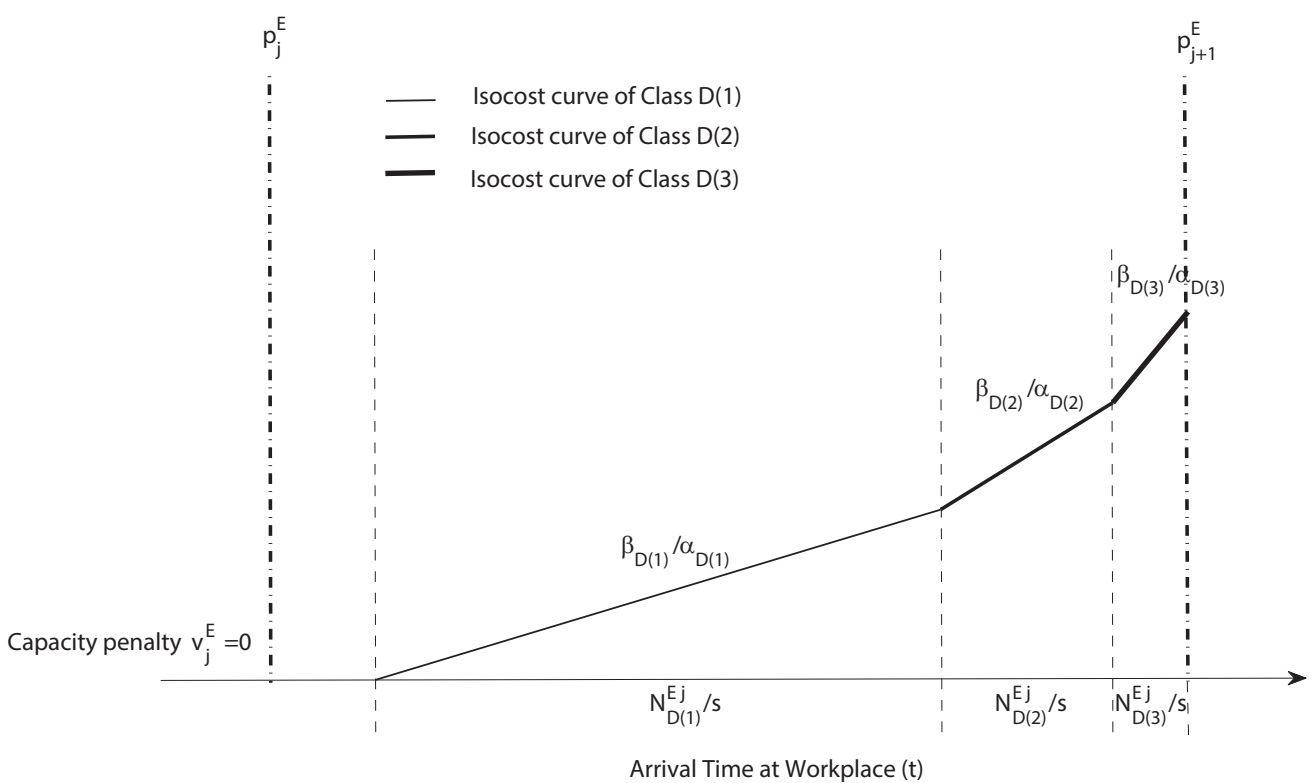

(a) With Idle Time (Unconstrained)

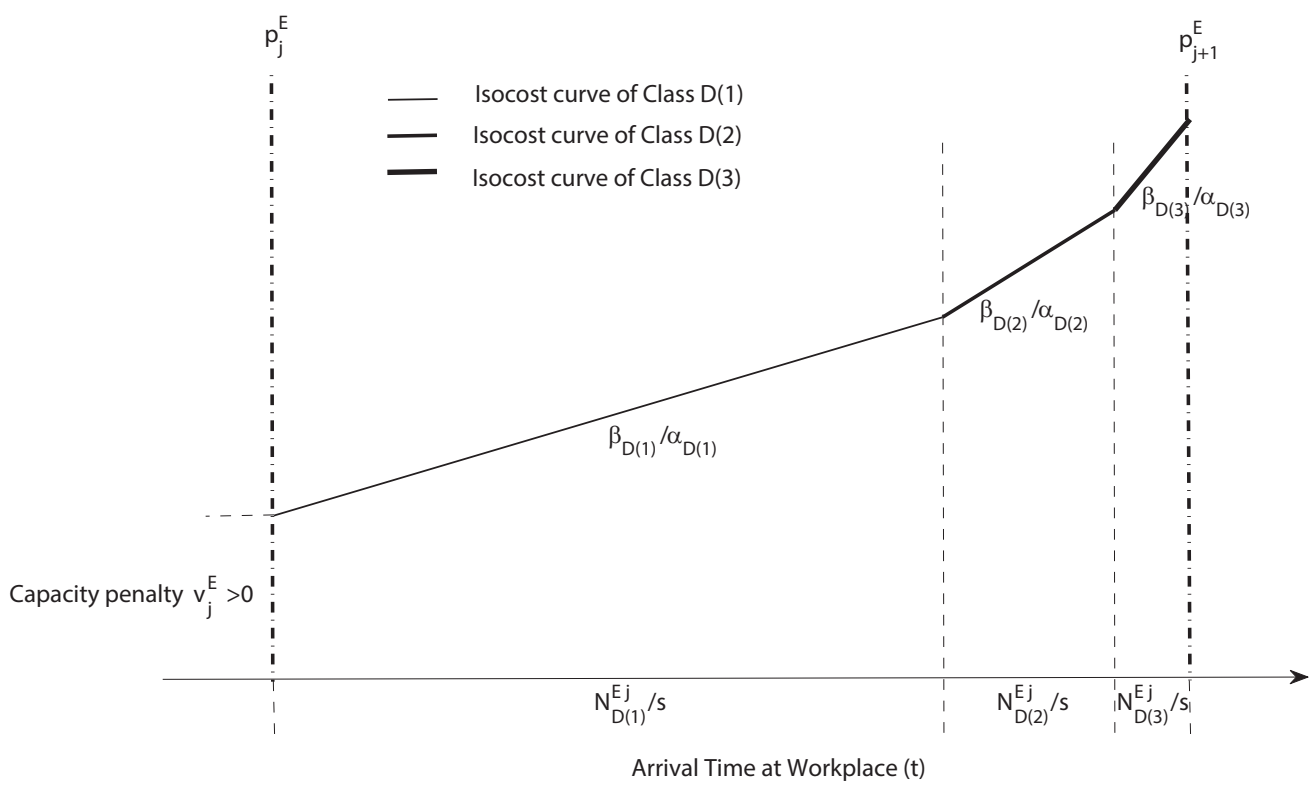

(b) Without Idle Time (Constrained)

Figure 3: Illustration of user equilibrium in an arrival window 
delay $\left(T_{i}^{A_{j}}\right)$ and schedule delay $\left(t^{*}-t_{i}^{E_{j}}\right.$ or $\left.t_{L(i)}^{L_{j}}-t^{*}\right)$, can be written as follows:

$$
\begin{array}{rlrl}
T_{i}^{E_{j}} & =\sum_{k=1}^{i-1} \frac{\beta_{k}}{\alpha_{k}} \frac{N_{k}^{E_{j}}}{s}+v_{j}^{E}, & i=1,2, \ldots, n, j=0,1, \ldots, m^{E} ; \\
t^{*}-t_{i}^{E_{j}}=\sum_{k=i}^{n} \frac{N_{k}^{E_{j}}}{s}+p_{j+1}^{E}, & i=1,2, \ldots, n, j=0,1, \ldots, m^{E} ; \\
T_{L(i)}^{L_{j}}=\sum_{k=1}^{i-1} \frac{\gamma_{L(k)}}{\alpha_{L(k)}} \frac{N_{L(k)}^{L_{j}}}{s}+v_{j}^{L}, & i=1,2, \ldots, n, j=0,1, \ldots, m^{L} ; \\
t_{L(i)}^{L_{j}}-t^{*} & =\sum_{k=i}^{n} \frac{N_{L(k)}^{L_{j}}}{s}+p_{j+1}^{L}, & i=1,2, \ldots, n, j=0,1, \ldots, m^{L} .
\end{array}
$$

Excluding the extra delay associated with the capacity constraints, the "actual" travel cost $c_{i}^{A_{j}}$ can be written as:

$$
\begin{aligned}
c_{i}^{E_{j}} & =\alpha_{i} T_{i}^{E_{j}}+\beta_{i}\left(t^{*}-t_{i}^{E_{j}}\right)+\pi_{j}^{E} \\
& =\alpha_{i} \sum_{k=1}^{i-1} \frac{\beta_{k}}{\alpha_{k}} \frac{N_{k}^{E_{j}}}{s}+\beta_{i} \sum_{k=i}^{n} \frac{N_{k}^{E_{j}}}{s}+\beta_{i} p_{j+1}^{E}+\pi_{j}^{E}, \quad i=1,2, \ldots, n, j=0,1, \ldots, m^{E} ; \\
c_{L(i)}^{L_{j}} & =\alpha_{L(i)} T_{L(i)}^{L_{j}}+\gamma_{L(i)}\left(t_{L(i)}^{L_{j}}-t^{*}\right)+\pi_{j}^{L} \\
& =\alpha_{L(i)} \sum_{k=1}^{i-1} \frac{\gamma_{L(k)}}{\alpha_{L(k)}} \frac{N_{L(k)}^{L_{j}}}{s}+\gamma_{L(i)} \sum_{k=i}^{n} \frac{N_{L(k)}^{L_{j}}}{s}+\gamma_{L(i)} p_{j+1}^{L}+\pi_{j}^{L}, \quad i=1,2, \ldots, n, j=0,1, \ldots, m^{L},
\end{aligned}
$$

Define the actual travel cost measured in the unit of travel time as

$$
C_{i}^{A_{j}} \equiv \frac{c_{i}^{A_{j}}}{\alpha_{i}}, \quad i=1,2, \ldots, n, j=0,1, \ldots, m^{A}, A=E, L .
$$

Then, UE conditions across arrival windows can be represented as follows:

$$
\begin{aligned}
& \left(C_{i}^{A_{j}}+v_{j}^{A}-\mu_{i}\right) N_{i}^{A_{j}}=0, \\
& C_{i}^{A_{j}}+v_{j}^{A}-\mu_{i} \geq 0, \\
& N_{i}^{A_{j}} \geq 0
\end{aligned}
$$$$
i=1,2, \ldots, n, j=0,1, \ldots, m^{A}, A=E, L
$$

That is, if class $i$ has positive flows in arrival window $A_{j}$, its corresponding travel cost $\left(C_{i}^{A_{j}}+v_{j}^{A}\right)$ must be the minimum among all windows. Moreover, the demand conservation and nonnegativity conditions are given by

$$
\begin{array}{lr}
\sum_{A \in\{E, L\}} \sum_{j=0}^{m^{A}} N_{i}^{A_{j}}=N_{i}, & i=1,2, \ldots, n ; \\
N_{i}^{A_{j}} \geq 0, & i=1,2, \ldots, n ;, j=0,1, \ldots, m^{A} ; A=E, L
\end{array}
$$

Let

$$
\boldsymbol{\Omega}=\{\mathbf{N} \mid \mathbf{N} \text { satisfies Constraints }(10 a) \text { and }(10 b)\}
$$


and

$$
\Sigma=\{\mathbf{N} \mid \mathbf{N} \text { satisfies Constraints }(5 b)\}
$$

Then, the traffic assignment problem depicted in Figure 2 can be formulated as a variational inequality problem. The result below follows directly from Liu et al. (2015) (the proof is omitted here for brevity).

Proposition 1. A route flow pattern $\mathbf{N}^{*} \in \Omega \cap \Sigma$ is a user equilibrium solution of the equivalent static traffic assignment problem if and only if it solves the variational inequalities problem $\operatorname{VIP}(\boldsymbol{\Omega} \cap \mathbf{\Sigma}, \mathbf{C})$ :

$$
\left\langle\mathbf{C}\left(\mathbf{N}^{*}\right)^{T}, \mathbf{N}-\mathbf{N}^{*}\right\rangle \geq 0
$$

where $\mathbf{C}$ are defined in Equation (8).

Remark: Unlike Liu et al. (2015) (which does not consider step toll), the above VIP formulation requires that the general cost be measured in the unit of travel time. This requirement is derived from the fact that the multiplier associated with the capacity constraint must be identical for all classes. Clearly, only if the multiplier itself is measured in the unit of time can this requirement be fulfilled. Accordingly, all the cost items in the complementarity conditions (9) must also be in the unit of time.

Proposition 2 (Solution existence). There exists a solution to $\operatorname{VIP}(\boldsymbol{\Omega} \cap \mathbf{\Sigma}, \mathbf{C})$ as defined in (11), if (1) $N_{i} \geq 0, \forall i$ and (2) $\beta_{i}<\alpha_{i}, \forall i$.

Proof: Note that $\Omega \cap \Sigma$ is a nonempty polyhedral. It is nonempty because of condition (1) and the fact that not all routes are constrained; and it is a polyhedral because all constraints are affine. Thus, the solution existence easily follows from Proposition 1 of Liu et al. (2015). Note that Condition (2) is required to ensure the validity of the UE conditions within each window (e.g. Lindsey, 2004).

Proposition 3 (Solution uniqueness). The problem $\operatorname{VIP}(\Omega \cap \Sigma, \mathbf{C})$ defined in (11) has a unique solution if (1) there exists at least one solution and (2) $\beta_{i} / \alpha_{i} \neq \beta_{j} / \alpha_{j}, \gamma_{i} / \alpha_{i} \neq \gamma_{j} / \alpha_{j}, \forall i \neq j$.

Proof: Note that the Jacobian matrix $\mathbf{J}$ of $\mathbf{C}$ is in the form of

$$
\mathbf{J}=\left(\begin{array}{ccccc}
J_{E} & & & & \\
& J_{E} & & & \\
& & \ddots & & \\
& & & J_{L} & \\
& & & & J_{L}
\end{array}\right)
$$

There are $\left(m^{E}+1\right)$ matrices $J_{E}$ and $\left(m^{L}+1\right)$ matrices $J_{L}$ in the Jacobian matrix $\mathbf{J}$, and

$$
J_{E}=\frac{1}{S}\left(\begin{array}{cccc}
\frac{\beta_{E(1)}}{\alpha_{E(1)}} & \frac{\beta_{E(1)}}{\alpha_{E(1)}} & \cdots & \frac{\beta_{E(1)}}{\alpha_{E(1)}} \\
\frac{\beta_{E(1)}}{\alpha_{E(1)}} & \frac{\beta_{E(2)}}{\alpha_{E(2)}} & \cdots & \frac{\beta_{E(2)}}{\alpha_{E(2)}} \\
\vdots & \vdots & \ddots & \vdots \\
\frac{\beta_{E(1)}}{\alpha_{E(1)}} & \frac{\beta_{E(2)}}{\alpha_{E(2)}} & \cdots & \frac{\beta_{E(n)}}{\alpha_{E(n)}}
\end{array}\right), J_{L}=\frac{1}{S}\left(\begin{array}{cccc}
\frac{\gamma_{L(1)}}{\alpha_{L(1)}} & \frac{\gamma_{L(1)}}{\alpha_{L(1)}} & \cdots & \frac{\gamma_{L(1)}}{\alpha_{L(1)}} \\
\frac{\gamma_{L(1)}}{\alpha_{L(1)}} & \frac{\gamma_{L(2)}}{\alpha_{L(2)}} & \cdots & \frac{\gamma_{L(2)}}{\alpha_{L(2)}} \\
\vdots & \vdots & \ddots & \vdots \\
\frac{\gamma_{L(1)}}{\alpha_{L(1)}} & \frac{\gamma_{L(2)}}{\alpha_{L(2)}} & \cdots & \frac{\gamma_{L(n)}}{\alpha_{L(n)}}
\end{array}\right)
$$


The structure of $\mathbf{J}$ and the $\mathbf{C}$ is identical to that from the multi-route model in Liu et al. (2015). Hence solution uniqueness follows from Proposition 4 (Liu et al., 2015).

Remark. It is worth noting that Proposition 3 only establishes the uniqueness of the UE flow pattern. Lindsey (2004) pointed out that the UE cost $\mu$ may not be unique with the step tolls, since some classes may be able to "shift (their isocost curve) up or down slightly" without affecting other classes. This is expected since $\mu$ includes the multipliers for the capacity constraints, which may not be unique. Appendix B demonstrates this issue using a small example.

\subsection{Solution algorithm}

In this section, an algorithm is developed to solve the VIP formulation. To handle the capacity constraints, the algorithm consists of two loops. The inner loop solves an unconstrained UE problem with fixed multipliers associated with capacity constraints. The outer loop adjusts the multipliers according to the saturation status of the capacity constraints. We note that Liu et al. (2015) solve their problem using an algorithmic idea similar to what is adopted in our inner loop, but little details of their algorithm were reported.

In the inner loop, the unconstrained UE problem is solved based on Gauss-Seidel decomposition. The idea is to equilibrate the flows of only one class (or O-D pair as in Figure 2) at a time, while fixing the flows of all other classes. This equilibration is performed sequentially for each class and repeated until a desired convergence measure is achieved. According to Equation (8), if the multiplier and the flows of all other classes are known, the cost functions for a given class can be written as a one-dimensional linear function of flows $N_{i}^{A_{j}}$

$$
\begin{aligned}
C_{i}^{E_{j}} & =\frac{\beta_{i}}{\alpha_{i}} \frac{N_{i}^{E_{j}}}{s}+\sum_{k=1}^{i-1} \frac{\beta_{k}}{\alpha_{k}} \frac{N_{k}^{E_{j}}}{s}+\frac{\beta_{i}}{\alpha_{i}} \sum_{k=i+1}^{n} \frac{N_{k}^{E_{j}}}{s}+\frac{\beta_{i}}{\alpha_{i}} p_{j+1}^{E}+\frac{\pi_{j}^{E}}{\alpha_{i}}+v_{j}^{E} \\
& ={ }^{d e f} e_{i}^{E_{j}} N_{i}^{E_{j}}+f_{i}^{E_{j}}, i=1,2, \ldots, n, j=0,1, \ldots, m^{E} ; \\
C_{L(i)}^{L_{j}} & =\frac{\gamma_{L(i)}}{\alpha_{L(i)}} \frac{N_{L(i)}^{L_{j}}}{s}+\sum_{k=1}^{i-1} \frac{\gamma_{L(k)}}{\alpha_{L(k)}} \frac{N_{L(k)}^{L_{j}}}{s}+\frac{\gamma_{L(i)}}{\alpha_{L(i)}} \sum_{k=i+1}^{n} \frac{N_{L(k)}^{L_{j}}}{s}+\frac{\gamma_{L(i)}}{\alpha_{L(i)}} p_{j+1}^{L}+\frac{\pi_{j}^{L}}{\alpha_{L(i)}}+v_{j}^{L} \\
& ={ }^{d e f} e_{L(i)}^{L_{j}} N_{L(i)}^{L_{j}}+f_{L(i)}^{L_{j}}, i=1,2, \ldots, n, j=0,1, \ldots, m^{L} ;
\end{aligned}
$$

where $e_{i}^{A_{j}}$ and $f_{i}^{A_{j}}$ are the slopes and intercepts, respectively. Such a basic traffic assignment problem with linear cost functions is easy to solve. Algorithm 1 details a procedure, which first ranks the intercepts of the cost functions, and then solves a series of linear equation systems until the UE conditions are satisfied.

After solving the unconstrained UE solution, the total flow assigned to each arrival window $A_{j}$, i.e., $N^{A_{j}}$, is compared against the capacity $s\left(p_{A}^{j}-p_{A}^{j+1}\right)$. If $N^{A_{j}}$ is larger than $s\left(p_{A}^{j}-p_{A}^{j+1}\right)$, the associated multiplier will be adjusted upward using the procedure listed from line 12 - 19 in Algorithm 2; otherwise, the multiplier will be adjusted downward using the procedure listed from line 20 - 25 in Algorithm 2. 


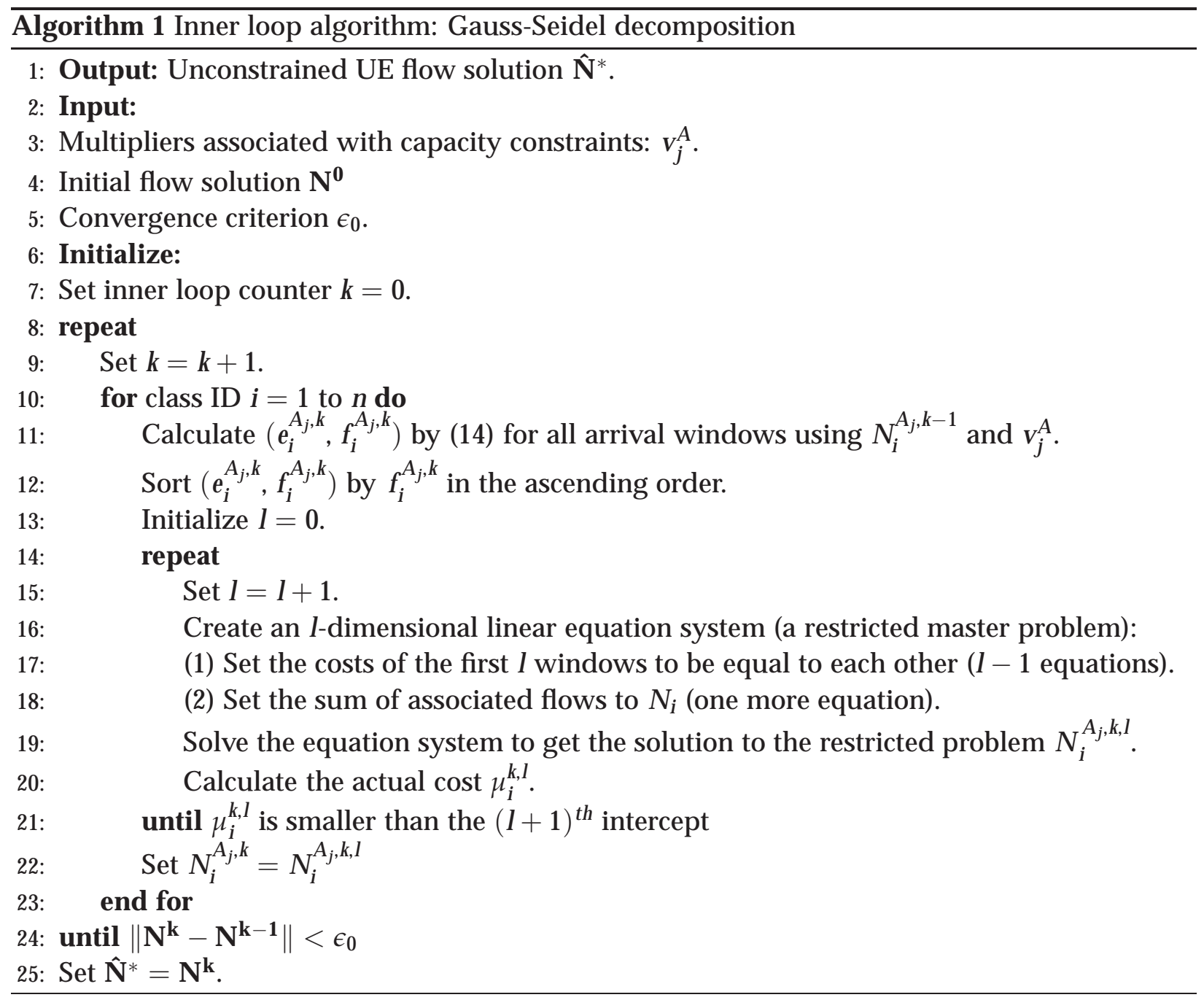




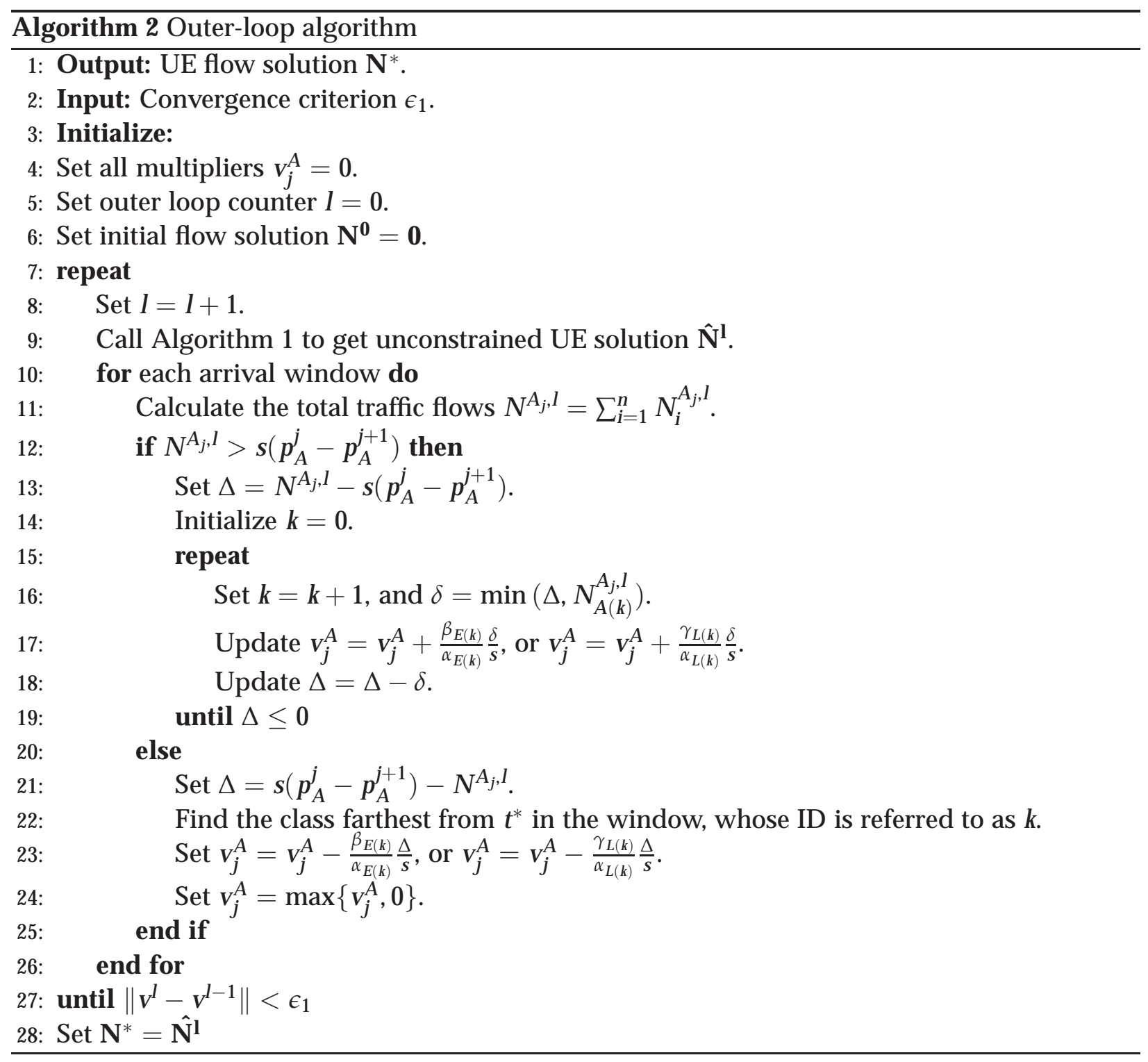




\section{Exact method}

In this section we describe a new method that analyzes the step-tolled bottleneck problem from a quite different perspective. Unlike the semi-analytical method discussed in the previous section, this method promises to locate the "exact solution". Perhaps more importantly, the new method makes it possible to express the relationship between step toll parameters and the corresponding UE flows in an explicit form, which opens the door to more effective design of such toll schemes.

Let us first make a formal definition of a variable that describes the appearance of classes in the $\left(m^{E}+m^{L}+2\right)$ arrival windows defined by the step toll scheme. For each arrival window $A_{j}$, the set of all classes that pass the bottleneck during that window is called the Class Membership Set (CMS) of the window, denoted as $\rho_{j}^{A}$. Note that $\rho_{j}^{A} \subseteq\{0,1,2, \ldots n\}$, where 1 to $n$ are the indexes of real classes and 0 is a dummy user class corresponding to idle arrival time (in other words a time slot occupied by the dummy class is actually an idle slot). Note that for two untolled arrival windows, the dummy class is always present. Accordingly, we call a vector $\rho=\left\{\rho_{0}^{E}, \rho_{1}^{E}, \ldots, \rho_{m^{E}}^{E}, \rho_{m^{L}}^{L}, \ldots, \rho_{1}^{L}, \rho_{0}^{L}\right\}$ a Class Membership Set Realization (CMSR).

The basic idea behind the exact method arises from the concept of CMSR. For any given CMSR, the class-specific flows in each arrival window can be written analytically as functions of (unknown) class-specific UE costs (see Section 4.1 for details). The vector of UE costs can then be solved from a linear equation system that simply links the class-specific flows in each arrival time to the total class flows. Finally, the UE cost vector solved from the above process must be checked to ensure that it satisfies the UE conditions implied by the CMSR (see Section 4.2 for details). If it does, then the solution obtained is confirmed to be an UE solution. Now, Proposition 2 and Proposition 3 state that there is one and only one UE solution. Since this unique UE solution must correspond to a unique CMSR, a brute-force solution method would be to enumerate all CMSRs and identify the "correct" one, i.e. satisfying all UE conditions. Because enumeration is clearly impractical, a heuristic method will be developed (see Section 4.3 for details).

\subsection{Find UE costs for a given CMSR}

The goal is to establish a linear relationship between the user demands $\left(N_{i}\right)$ and the UE costs $\left(\mu_{i}\right)$ for a given CMSR. We first define $n_{D}^{A_{j}}$ as the number of classes that present in arrival window $A_{j}$. Recall that $D^{A_{j}}(i)$ is the ratio rank ID of the class whose time inflexibility ranks at the $i^{\text {th }}$ place among all presenting classes in arrival window $A_{j}$.

By rearranging Equation (1) and setting $t^{*}=0$, the isocost curve functions (including the dummy class 0 ) can be written as

$$
T(t)=a_{i}^{A_{j}} t+b_{i}^{A_{j}} \quad i=0,1,2, \ldots, n, j=0,1, \ldots, m^{A}, A=E, L,
$$

where

$$
\begin{aligned}
& a_{i}^{A_{j}}=\left\{\begin{array}{cc}
\frac{\beta_{i}}{\alpha_{i}} & i=1,2, \ldots, n, j=0,1, \ldots, m^{E} \\
-\frac{\gamma_{i}}{\alpha_{i}} & i=1,2, \ldots, n, j=0,1, \ldots, m^{L} \\
0 & i=0, j=0,1, \ldots, m^{A}, A=E, L
\end{array} ;\right. \\
& b_{i}^{A_{j}}=\left\{\begin{array}{cc}
\mu_{i}-\frac{\pi_{j}^{A}}{\alpha_{i}} & i=1,2, \ldots, n, j=0,1, \ldots, m^{A}, A=E, L \\
0 & i=0, j=0,1, \ldots, m^{A}, A=E, L
\end{array}\right.
\end{aligned}
$$




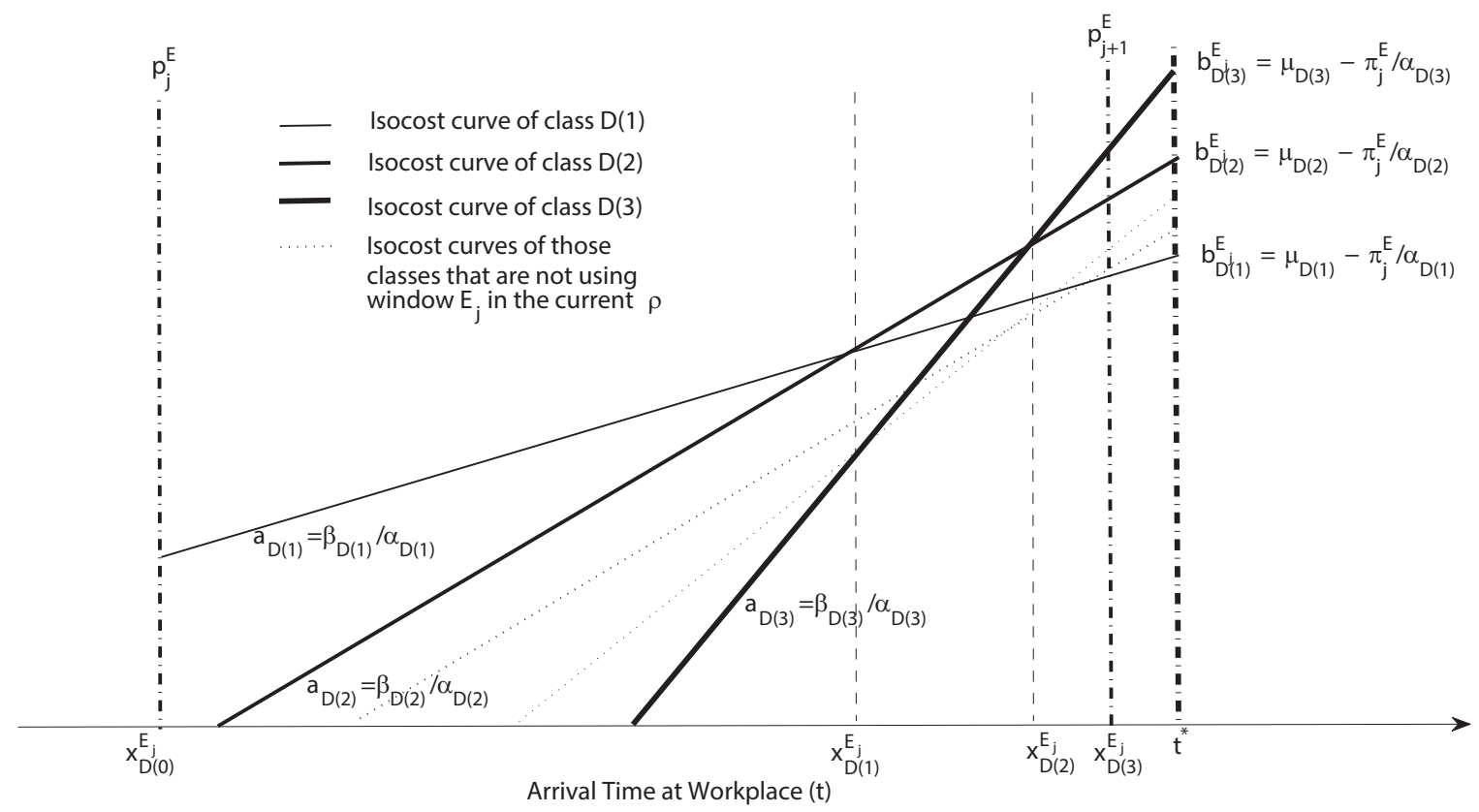

Figure 4: Illustration of isocost curves in the arrival window $E_{j}$

where $\mu_{i}$ is the UE cost of class $i$. Note that, since the dummy class 0 will occupy any time slot for which no real user has a positive willingness to pay, their isocost curves can be simply set as $T(t)=0$. Evidently, the time inflexibility ratio for class 0 will be ranked lowest among all classes. Hence, the ranked ID for the dummy class $A(0)=0(A=E, L)$.

For a given CMSR $\rho$, the departure order of all classes is known for every arrival window. Consequently, the flows of these classes in that window can be expressed as a function of $\mu_{i}, \forall i$. Figure 4 provides an illustrative example, where three classes out of five appear in window $E_{j}$ for a given $\rho$. Clearly, the smaller is the time inflexibility ratio, the farther away from $t^{*}$ will the class be located. Define $x_{A\left(D^{\left.A_{j}(i)\right)}\right.}^{A_{j}}$ as the horizontal coordinate for the farthest-from- $t^{*}$ user of the presenting class that has the $i^{\text {th }}$ lowest time inflexibility ratio among all presenting classes, in the arrival window $A_{j}$. Then, using the isocost curves in Equation (15), they can be written as

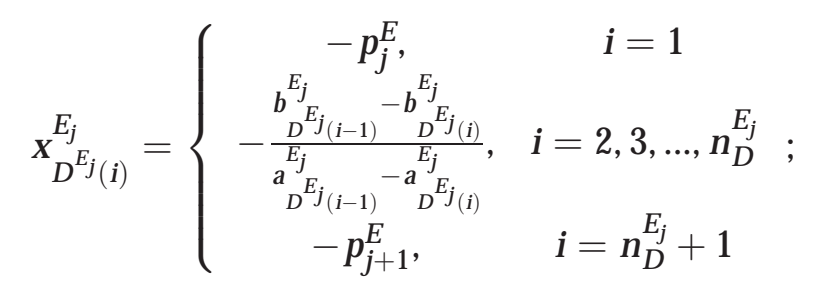

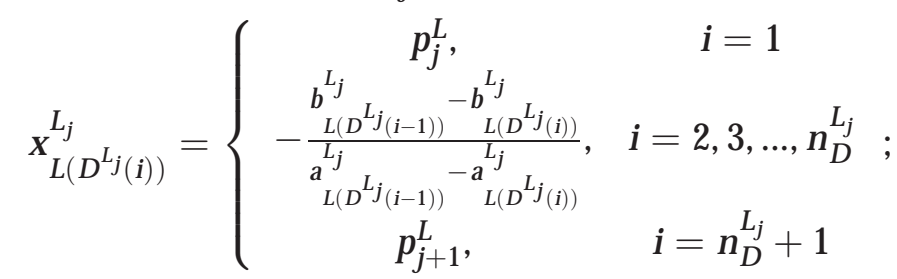

where $x_{A\left(D^{A_{j}}(1)\right)}^{A_{j}}$ and $x_{A\left(D^{A_{j}}\left(n_{D}^{A_{j}}+1\right)\right)}^{A_{j}}(A=E, L)$ represent the boundaries of the arrival window $A_{j}$. 
Once the above coordinates are located, the amount of flows for each class of commuters in $A_{j}$ can be computed as

$$
N_{A\left(D^{\left.A_{j}(i)\right)}\right.}^{A_{j}}=s\left(x_{A\left(D^{\left.A_{j}(i+1)\right)}\right.}^{A_{j}}-x_{A\left(D^{\left.A_{j}(i)\right)}\right.}^{A_{j}}\right), i=1,2, \ldots, n_{D}^{A_{j}} .
$$

Note that the classes that do not appear in the window for the given $\rho$ would have zero flows. Equations (16-18) together represent class-specific flows in each arrival window as functions of the UE travel cost vector $\mu$. Specifically, since $b_{i}^{A_{j}}$ are linear combinations of $\mu$, and $a_{i}^{A_{j}}$ are constant, $x_{A\left(D^{A_{j}}(i)\right)}^{A_{j}}$ are in fact linear functions of $\mu$. Finally, the demand conservation conditions of each class dictates that

$$
\sum_{A \in\{E, L\}} \sum_{j=0}^{m^{A}} N_{i}^{A_{j}}=N_{i}, \quad i=1,2, \ldots, n .
$$

(19) is a $n \times n$ linear equation system that has $\mu$ as the unknowns. While we expect the system usually has a unique solution, the possibility of having a singular system cannot be ruled out. However, such cases needs not concern us because a CMSR corresponding to a singular (19) cannot be the UE solution and hence can be simply discarded.

\subsection{Check the validity of a solution corresponding to a given CMSR}

The solution given by the linear equation system (19) is valid only if it produces isocost curves consistent with the same CMSR that leads to (19). This consistency check can be performed by examining several constraints, as explained below.

First, for any class that does appear in $A_{j}$, its flow defined in (18) must be nonnegative, which leads to the following constraints:

$$
x_{A\left(D^{\left.A_{j}(i+1)\right)}\right.}^{A_{j}} \geq x_{A\left(D^{\left.A_{j}(i)\right)}\right.}^{A_{j}}, i=1,2, \ldots, n_{D}^{A_{j}} .
$$

Second, for any class that does not appear in $A_{j}$, we must ensure that its isocost curve is "dominated" by those of the classes that do appear. We classify these "missing classes" into three categories.

For those whose $\left|a_{k}^{A}\right|$ (the absolute value of the slope of the isocost curve) are smaller than $\left|a_{A\left(D^{\left.A_{j}(1)\right)}\right.}^{A}\right|$, the vertical intercept of their isocost curves at the far end of the arrival window must be lower than that of $A\left(D^{A_{j}}(1)\right)$, i.e.

$$
\begin{array}{ll}
b_{E\left(D^{\left.E_{j}(1)\right)}\right.}^{E_{j}}-a_{E\left(D^{\left.E_{j}(1)\right)}\right.}^{E_{j}} p_{j}^{E} \geq b_{k}^{E_{j}}-a_{k}^{E_{j}} p_{j}^{E}, & k=0, E(1), \ldots, E\left(D^{E_{j}}(1)-1\right) ; \\
b_{L\left(D^{\left.L_{j}(1)\right)}\right.}^{L_{j}}+a_{L\left(D^{\left.L_{j}(1)\right)}\right.}^{L_{j}} p_{j}^{L} \geq b_{k}^{L_{j}}+a_{k}^{L_{j}} p_{j}^{L}, & k=0, L(1), \ldots, L\left(D^{L_{j}}(1)-1\right) .
\end{array}
$$

For the missing classes whose $\left|a_{k}^{A}\right|$ satisfies the following condition

$$
\left|a_{A\left(D^{\left.A_{j}(i)\right)}\right.}^{A}\right|<\left|a_{k}^{A}\right|<\left|a_{A\left(D^{\left.A_{j}(i+1)\right)}\right.}^{A}\right|,
$$


the intersection of its isocost curve with that of $A\left(D^{A_{j}}(i+1)\right)$ must lie further away from $t^{*}$ compared to the intersection between isocost curves of $A\left(D^{A_{j}}(i)\right)$ and $A\left(D^{A_{j}}(i+1)\right)$. Mathematically this requirement can be written as follows:

$$
\begin{aligned}
& x_{E\left(D^{\left.E_{j}(i+1)\right)}\right.}^{E_{j}}\left(a_{E\left(D^{\left.E_{j}(i)\right)}\right.}^{E_{j}}-a_{k}^{E_{j}}\right) \geq b_{E\left(D^{\left.E_{j}(i)\right)}\right.}^{E_{j}}-b_{k}^{E_{j}}, \quad i=1,2, \ldots, n_{D}^{E_{j}}-1, k=E\left(D^{E_{j}}(i)+1\right), \ldots, E\left(D^{E_{j}}(i+1)-1\right) ; \\
& x_{L\left(D^{\left.L_{j}(i+1)\right)}\right.}^{L_{j}}\left(a_{L\left(D^{\left.L_{j}(i)\right)}\right.}^{L_{j}}-a_{k}^{L_{j}}\right) \leq b_{L\left(D^{\left.L_{j}(i)\right)}\right.}^{L_{j}}-b_{k}^{L_{j}}, \quad i=1,2, \ldots, n_{D}^{L_{j}}-1, k=L\left(D^{L_{j}}(i)+1\right), \ldots, L\left(D^{L_{j}}(i+1)-1\right) .
\end{aligned}
$$

Finally, for all the missing classes whose $\left|a_{k}^{A}\right|>\left|a_{A\left(D^{A_{j}\left(n_{D}\right)}{ }_{A_{j}}^{A}\right.}\right|$, the vertical intercept of its isocost curve with the boundary of the arrival windows near to $t^{*}$ must be lower than that of $A\left(D^{A_{j}}\left(n_{D}^{A_{j}}\right)\right)$ :

$$
\begin{array}{ll}
b_{E\left(D^{\left.E_{j}\left(n_{D}^{E_{j}}\right)\right)}\right.}^{E_{j}}-a_{E\left(D^{\left.E_{j}\left(n_{D} E_{j}\right)\right)}\right.}^{E_{j}} p_{j+1}^{E} \geq b_{k}^{E_{j}}-a_{k}^{E_{j}} p_{j+1}^{E}, & k=E\left(D^{E_{j}}\left(n_{D}^{E_{j}}\right)+1\right), \ldots, n ; \\
b^{L_{j}} D_{L\left(D^{\left.L_{j}\left(n_{D}^{L_{j}}\right)\right)}\right.}^{L_{j}}+a^{L_{j} D^{\left.L_{j}\left(n_{D}^{L_{j}}\right)\right)}} p_{j+1}^{L} \geq b_{k}^{L_{j}}+a_{k}^{L_{j}} p_{j+1}^{L}, & k=L\left(D^{L_{j}}\left(n_{D}^{L_{j}}\right)+1\right), \ldots, n .
\end{array}
$$

Note that the total number of constraints defined by (20-23) equals $n$, the number of classes. These $n$ constraints can be easily validated once the UE cost vector $\mu$ is obtained from the linear equation system (18). If they are all satisfied, we conclude that the flow pattern associated with the CMSR is indeed the UE solution.

\subsection{Solution algorithm}

Having explained how to check whether or not a given CMSR satisfies all UE conditions, we proceed to develop a procedure to identify the "correct" CMSR. The most straightforward approach is to enumerate all possible CMSRs, since their number is finite. Algorithm 3 formally describes the procedure.

The complexity of the above algorithm depends on the maximum number of CMSR $K$. We now analyze how $K$ is related to the size of the problem, i.e., the number of class $n$ and the number of toll steps $\left(m^{E}+m^{L}\right)$. For the two untolled windows, there must be an idle arrival time. Hence, the dummy user class 0 will always be present, which means the size of possible CMSR for these period is $2^{n}$, The tolled windows, on the other hand, can have up to $(n+1)$ user classes (including the dummy user). This gives a total of $2^{(n+1)}-1$ possible combinations since at least one user class will be present. Combining all periods together, we can see that $K=4^{n}\left(2^{(n+1)}-1\right)^{\left(m^{E}+m^{L}\right)}$. Clearly, Algorithm 3 will soon become prohibitively time-consuming as $n$ and/or $\left(m^{E}+m^{L}\right)$ increases. In fact, a quick calculation indicates that for $n=10$ and $m^{E}+m^{L}=2, K$ will exceed one trillion.

To improve the computational efficiency, a heuristic method is developed below. The method is inspired by the premise that the solution verification step (line 8) in Algorithm 3 may be used to guide the search for the correct CMSR. To see this, first note that there is one and only one class corresponding to each of the CMSR's flow constraints (20), (21), (22), and (23). Hence, if a constraint is violated, the appearance state of its corresponding class may need to be flipped. 


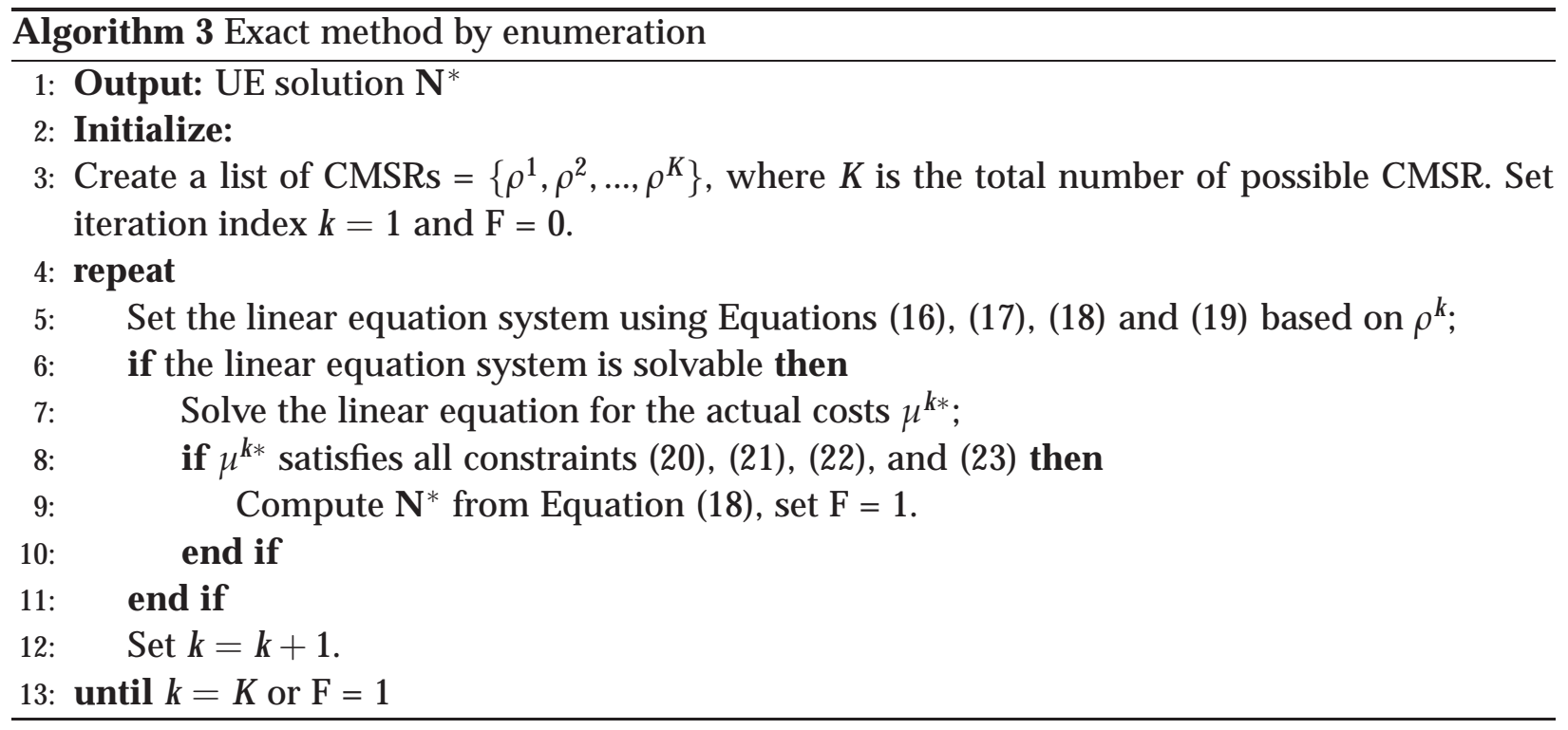

This idea gives rise to the following procedure, which starts by choosing a predefined CMSR (e.g. one that places all commuter classes in every arrival period). In each iteration, we identify the constraint subject to the most severe violation in each arrival time, and then flip the appearance status of its corresponding class. The new generated CMSR is then tested in the next iteration until no change in CMSR is needed (which implies that the solution is at UE). It turns out that this seemingly simple idea produces exceedingly satisfactory results. Details of the heuristic method can be found in Algorithm 4.

\section{Numerical examples}

Our numerical experiments consist of a small example and a large example. The small example is used to demonstrate the procedures in details and explore how user heterogeneity might affect the optimal design of step tolls. The large example, which includes up to 1000 classes, is designed to test the computational efficiency of the semi-analytical and exact methods. All algorithms are coded in $\mathrm{C}++$ and tested on a laptop computer with an Intel(R) Core (TM) i7-2720QM CPU (2.20-3.30 GHz) and 8GB DDR3 1333 RAM.

The total system cost used in this section is defined as the total commuter cost excluding the toll revenue, in monetary values:

$$
S C=\sum_{i=1}^{n}\left[\alpha_{i} \mu_{i} N_{i}-\sum_{A \in\{E, L\}} \sum_{j=0}^{m^{A}} \pi_{j}^{A} N_{i}^{A_{j}}\right],
$$

where $\mu_{i}$ and $N_{i}^{A_{j}}$ are class-specific UE costs and flows.

\subsection{Two-class with a single-step-toll}

In this example, the bottleneck has a capacity $s=1000$ vehicle per hour (vph). There are two classes of commuters. The first class not only have a higher value of time, but also like to be on 


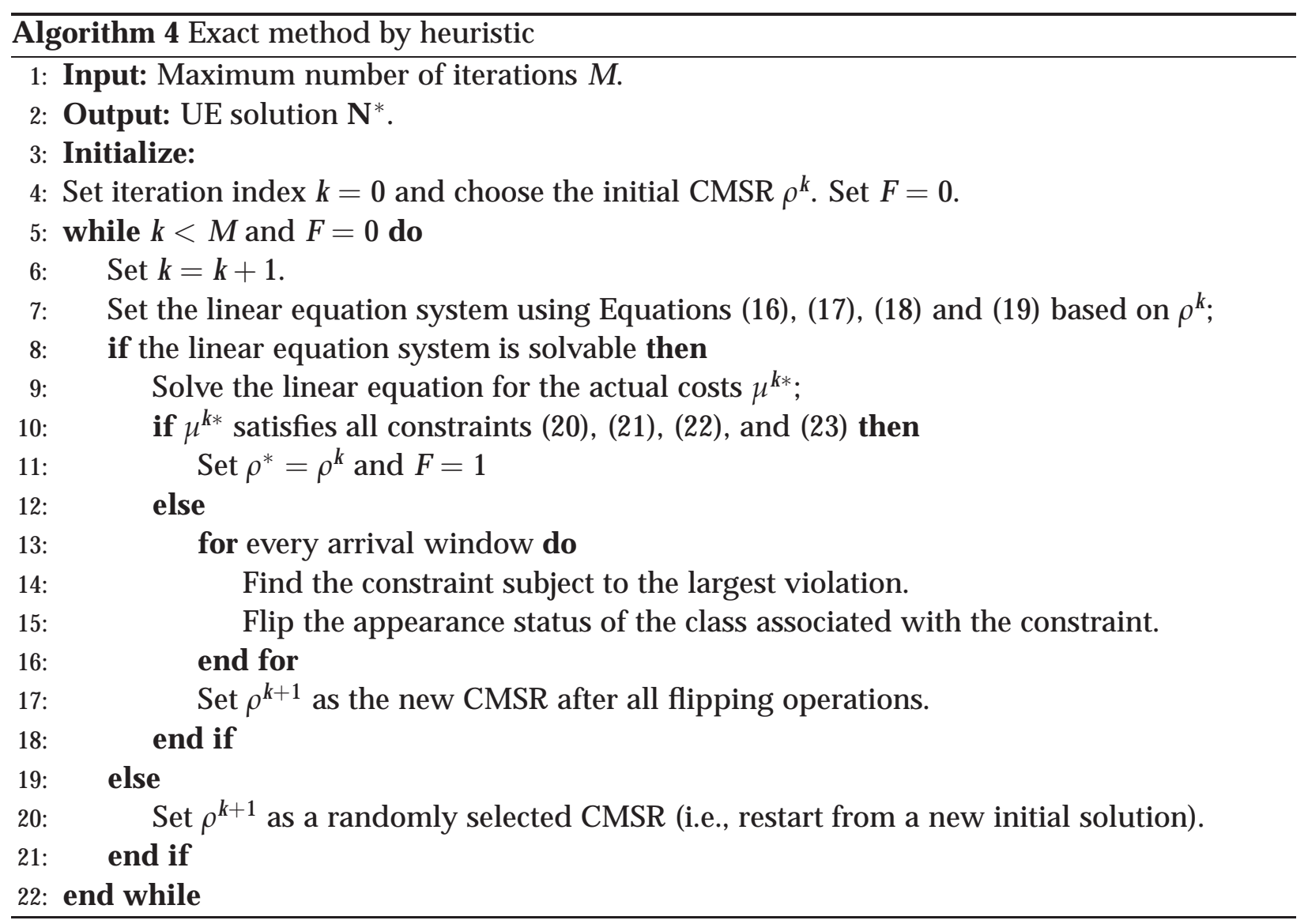


time, hence they have a low early arrival time inflexibility and high late arrival time inflexibility. The second class, on the other hand, have a lower value of time but would rather come to work late (i.e. a relatively low late arrival time inflexibility), than to be early at the workplace (i.e. a high early arrival time inflexibility). The parameters of two user classes are listed in Table 1. The last row in the table reports the average values of these parameters, which mimics the situation where the users cannot be differentiated, and hence must be treated as if there is only one aggregated class with its behavioral parameters being the average of the population.

Table 1: Travel preference and demands in the two-classes example

\begin{tabular}{c|c|ccc|cc}
\hline Class No. & Demand & \multicolumn{3}{|c|}{ Values of time } & \multicolumn{2}{c}{ Time inflexibility } \\
$i$ & $N_{i}$ & $\alpha_{i}(\$ /$ hour $)$ & $\beta_{i}$ (\$/hour) & $\gamma_{i}(\$ /$ hour $)$ & $\frac{\beta_{i}}{\alpha_{i}}$ & $\frac{\gamma_{i}}{\alpha_{i}}$ \\
\hline 1 & 5000 & 12 & 4 & 30 & $1 / 3$ & $5 / 2$ \\
2 & 5000 & 8 & 6 & 10 & $3 / 4$ & $5 / 4$ \\
Aggregated & 10000 & 10 & 5 & 20 & $1 / 2$ & 2 \\
\hline
\end{tabular}

We first find the system optimal single step toll for the "hypothetical" aggregated case, which can be solved analytically as $\left(p_{1}^{E}, \pi_{1}^{E}, p_{1}^{L}, \pi_{1}^{L}\right)=(4,20,1,20)$ (Laih, 1994). In words, the optimal toll is $\$ 20$, the start time of the toll would be 4 hours prior to $t^{*}$, and the end time would be 1 hour after $t^{*}$. In theory, this system optimal toll can reduce the total system cost by $25 \%$ if the users are indeed homogeneous with the aggregated parameters. In the following, this toll will then be used as the inputs for setting the two-class model. The objective is to examine its actual effect on heterogeneous users.

Appendix C explains in great details how the UE solutions are obtained using the two methods. A summary of results are reported in Table 2 and Figure 5 plots the isocost curves at UE for both the heterogeneous and homogeneous cases. First, we note that the homogeneous assump-

Table 2: Result information for the two-class with a single-step-toll example

\begin{tabular}{|c|c|c|c|c|c|c|c|c|c|}
\hline \multirow{3}{*}{$\begin{array}{c}\text { Class } \\
\text { Information }\end{array}$} & \multicolumn{4}{|c|}{ No-toll UE } & \multicolumn{4}{|c|}{ Tolled UE } & Total Cost \\
\hline & Total & Ser & Ana. & Heu. & Total & Sen & Ana. & Heu. & Reduced \\
\hline & Cost (\$) & Inner & Outer & Iter. & Cost (\$) & Inner & Outer & Iter. & By Toll \\
\hline Two Clas & 281250 & 4 & 1 & 2 & 245909 & 177 & 17 & 3 & $12.6 \%$ \\
\hline Aggregated & 400000 & 2 & 1 & 1 & 300000 & 2 & 1 & 1 & $25.0 \%$ \\
\hline
\end{tabular}

tion leads to severe overestimation in the overall system cost for both no-toll and tolled cases (by more than 30\%). Second, the system optimal toll of the homogeneous case only reduces the system cost by about $12.6 \%$ when user heterogeneity is recognized, instead of $25 \%$ as in the homogeneous case. Third and perhaps most important, the welfare effect of the toll is significantly altered. In the homogenous case, it is well known that the toll will reduce congestion at nobody's cost, i.e., all users will break even after the toll is implemented. Yet, Figure 5 ( $c^{*}$ reported in the plots) reveals that, with this toll both classes will be worse off (before the toll revenue is reimbursed). In fact, the second class's cost jumps more than $20 \%$ after the toll. This toll design has such surprisingly miserable welfare effects exactly because it is derived from the aggregated data and hence is sub-optimal for the actual problem. 


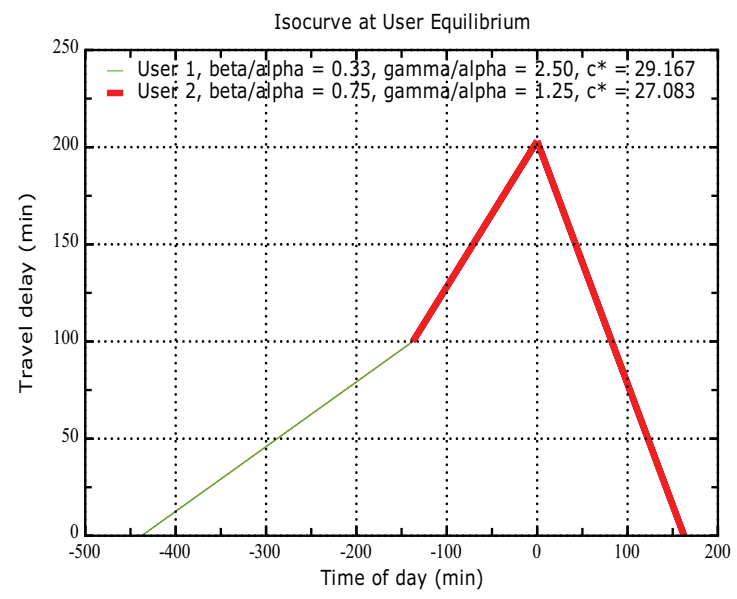

(a) No-toll UE, two classes

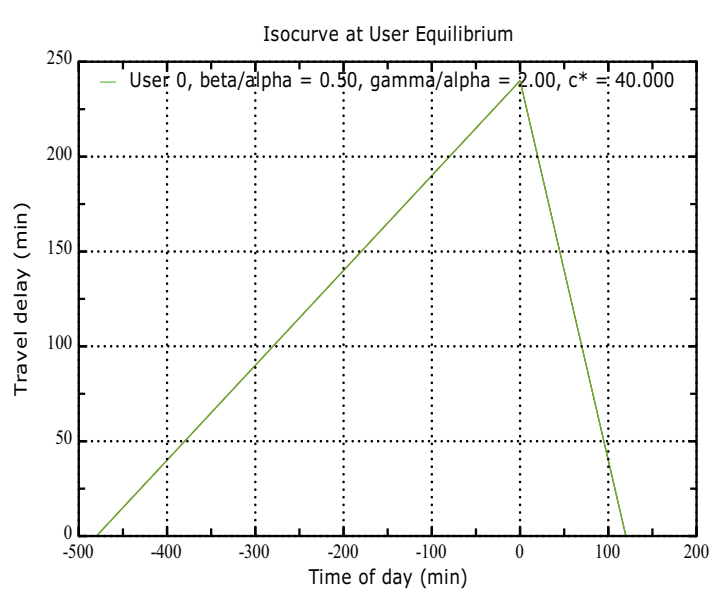

(c) No-toll UE, homogeneous assumption

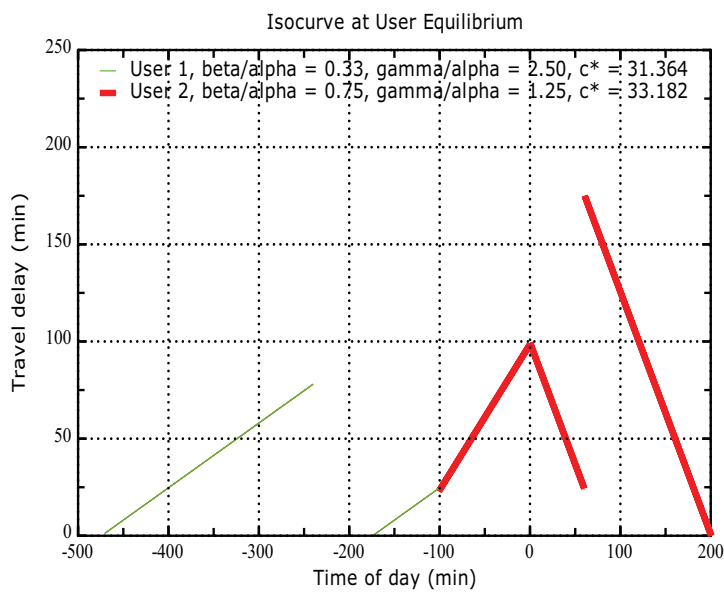

(b) Tolled UE, two classes

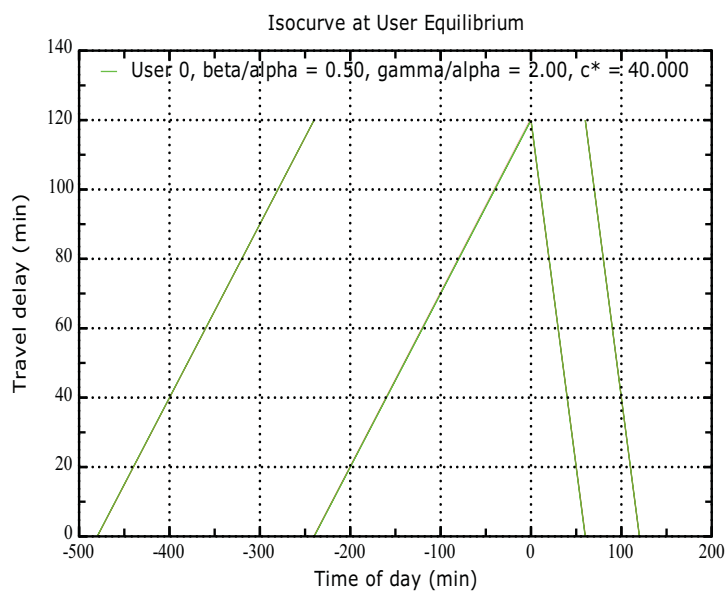

(d) Tolled UE, homogeneous assumption

Figure 5: Isocost curves for the two-class example with a single-step-toll

The above finding highlights the importance of recognizing user heterogeneity in the analysis and design of step tolls. We expect that a toll scheme designed with user heterogeneity in mind would outperforms the system optimal toll tested here. However, how to design such a toll is beyond the scope of the present paper and shall be addressed in subsequent studies.

\subsection{Experiment with larger examples}

The purpose of this experiment is to explore the computational aspects of the two methods proposed in this paper. In total 16 examples are tested, each with a different number of classes and/or a toll scheme.

The bottleneck capacity s is $1600 \mathrm{vph}$; the numbers of classes vary from 10, 50, 200, to 1000. We assume that: (1) the value of time $(\alpha)$ is uniformly distributed from 5 to 35 dollars per hour; (2) the early arrival time inflexibility $\left(\frac{\beta}{\alpha}\right)$ is uniformly distributed from 0.05 to 0.95 , independent of $\alpha$; (3) the late arrival time inflexibility $\left(\frac{\gamma}{\alpha}\right)$ is uniformly distributed from 1.05 to 3.95 , independent of $\alpha$ and $\frac{\beta}{\alpha}$; and (4) the total number of travelers are about 12000, so that the length of rush hour 
is about 7.5 hours. To avoid generating classes with very similar time inflexibility (which may affect the results), the sample is generated using the following procedure: (1) create $\alpha, \frac{\beta}{\alpha}$ and $\frac{\gamma}{\alpha}$ as arithmetic sequences from the lowest value to the highest; (2) rearrange the order of $\beta / \alpha$ and $\gamma / \alpha$ as random permutations to ensure independence; (3) generate the number of travelers for each class as an independent random variable following a Normal Distribution with mean $\frac{12000}{n}$ and standard deviation $\frac{1200}{n}$.

The four step toll schemes tested in this example are: (I) no-toll scheme; (II) a single-step-toll scheme from time -3 to 1 (hour), with a $\$ 20$ toll (written as $(-3,1, \$ 20)$ hereafter for simplicity); (III) a three-step-toll scheme with step tolls $(-4,-3, \$ 3),(-3,0, \$ 8)$ and $(0,1, \$ 5)$; (IV) a four-step-toll scheme of tolls $(-5,-2, \$ 10),(-2,0, \$ 20),(0,1, \$ 10)$, and $(1,2, \$ 5)$.

Table 3: Test results for the experiments with larger examples

\begin{tabular}{|c|c|c|c|c|c|c|}
\hline \multirow[b]{3}{*}{$n$} & \multicolumn{6}{|c|}{ Toll scheme I } \\
\hline & \multirow{2}{*}{$\begin{array}{l}\text { Total } \\
\text { Cost }\end{array}$} & \multicolumn{3}{|c|}{ Semi-Analytical } & \multicolumn{2}{|c|}{ Heuristic } \\
\hline & & Inner & Outer & Time & Iter. & Time \\
\hline 10 & 358734 & 202 & 1 & 0 m.sec. & 7 & $281 \mathrm{~m} . \mathrm{sec}$. \\
\hline 50 & 442119 & 57 & 1 & 0 m.sec. & 40 & $1.42 \mathrm{sec}$. \\
\hline 200 & 445029 & 1413 & 1 & 593 m.sec. & 158 & $7.21 \mathrm{sec}$. \\
\hline \multirow[t]{2}{*}{1000} & 440152 & 573 & 1 & $5.79 \mathrm{sec}$. & 794 & $14.9 \mathrm{~min}$. \\
\hline & \multicolumn{6}{|c|}{ Toll scheme II } \\
\hline \multirow{2}{*}{$\begin{array}{c}\text { Number of } \\
\text { Class }\end{array}$} & Total & \multicolumn{3}{|c|}{ Semi-Analytical } & \multicolumn{2}{|c|}{ Heuristic } \\
\hline & Cost & Inner & Outer & Time & Iter. & Time \\
\hline 10 & 270261 & 198 & 1 & $0 \mathrm{~m} . \mathrm{sec}$. & 11 & $452 \mathrm{~m} . \mathrm{sec}$. \\
\hline 50 & 327128 & 236 & 1 & $16.0 \mathrm{~m} . \mathrm{sec}$. & 55 & $1.97 \mathrm{sec}$. \\
\hline 200 & 327727 & 1098 & 1 & 982 m.sec. & 232 & 10.5 sec. \\
\hline \multirow[t]{2}{*}{1000} & 321655 & 295 & 1 & $6.01 \mathrm{sec}$. & 1117 & $21.2 \mathrm{~min}$. \\
\hline & \multicolumn{6}{|c|}{ Toll scheme III } \\
\hline \multirow{2}{*}{$\begin{array}{c}\text { Number of } \\
\text { Class }\end{array}$} & Total & \multicolumn{3}{|c|}{ Semi-Analytical } & \multicolumn{2}{|c|}{ Heuristic } \\
\hline & Cost & Inner & Outer & Time & Iter. & Time \\
\hline 10 & 280262 & 5746 & 52 & $47.0 \mathrm{~m} . \mathrm{sec}$. & 13 & 530 m.sec. \\
\hline 50 & 376199 & 1477 & 48 & 156 m.sec. & 70 & $2.52 \mathrm{sec}$ \\
\hline 200 & 373631 & 2810 & 54 & $3.06 \mathrm{sec}$. & 320 & $15.1 \mathrm{sec}$. \\
\hline \multirow[t]{2}{*}{1000} & 376136 & 14353 & 78 & $5.85 \mathrm{~min}$. & 1949 & $36.8 \mathrm{~min}$. \\
\hline & \multicolumn{6}{|c|}{ Toll scheme IV } \\
\hline \multirow{2}{*}{$\begin{array}{c}\text { Number of } \\
\text { Class }\end{array}$} & Total & \multicolumn{3}{|c|}{ Semi-Analytical } & \multicolumn{2}{|c|}{ Heuristic } \\
\hline & Cost & Inner & Outer & Time & Iter. & Time \\
\hline 10 & 271647 & 3093 & 28 & 31.0 m.sec. & 13 & 530 m.sec. \\
\hline 50 & 350633 & 6247 & 33 & 780 m.sec. & 78 & $2.84 \mathrm{sec}$ \\
\hline 200 & 348700 & 2921 & 35 & $3.90 \mathrm{sec}$. & 338 & $15.7 \mathrm{sec}$. \\
\hline 1000 & 343034 & 10730 & 39 & $5.32 \mathrm{~min}$. & 1834 & $33.0 \mathrm{~min}$. \\
\hline
\end{tabular}

Table 3 summarizes the computational performance of the two algorithms in solving the 16 examples. First, because we have used very tight convergence criterion for the semi-analytical method, we observed that both algorithms have consistently produced almost exactly same so- 
lution. Such a cross examination offer a strong proof that both algorithms are correctly designed and implemented.

As expected, the results show that the computation efforts required by both methods increase super-linearly with the number of classes. The semi-analytical method consistently outperforms the heuristic method by roughly an order of magnitude. Yet, considering the sheer space that the heuristic method has to search and the primitive nature of its design, the robust performance of the heuristic method seems a success. Moreover, as mentioned before, while it may not be the fastest in finding the step-tolled UE solution, it could provide a competitive alternative to the toll design problem.

Another noteworthy finding from the above table is that the performance of the semi-analytical method seems to be more sensitive to the toll schemes. For the same 1000-class example, the semi-analytical method spent 60 times as much time on solving toll scheme IV as it spent on solving toll scheme I. For the heuristic method, this difference is a little more than 2 times. The reason for the large discrepancy is that for both toll schemes I and II, the solution does not have binding capacity constraints, which effectively reduce the number of outer iterations for the semi-analytical method to 1. Evidently, the heuristic method cannot take this advantage. In fact, when some capacity constraints are binding, the difference in computational performance between the two methods seems relatively stable (toll schemes IIII and IV). We note that, however, the semi-analytical method can find a coarser solution at much less computational efforts than reported here.

\section{Conclusions}

In this paper we developed two numerical methods for solving the step-tolled user equilibrium problem for the bottleneck model with general user heterogeneity. The exact method is based on the idea of enumerating all possible departure orders until it identifies the one that produces the correct user equilibrium solution. While this method is only of theoretical interest, its heuristic version, which navigates through the solution space using the clue provided by "the feasibility cuts", offers a practically useful algorithm. The semi-analytical method, on the other hand, transforms the step-tolled user equilibrium problem into a static, asymmetric traffic assignment problem with side constraints, which is formulated as a variational inequality problem (VIP). This method allows us to derive existence and uniqueness results for the UE solution. Also, the special structure of the VIP makes it possible to derive very efficient assignment algorithm based on the notion of Gauss-Seidel decomposition.

These methods will enable researchers to solve the step-tolled bottleneck model with very general structure of user heterogeneity, which, to the best of our knowledge, has been an unresolved issue in the literature. Findings from our numerical experiments are summarized below.

- The proposed heuristic method, while primitive, successfully operationalizes the algorithmic idea behind the exact method. In all tested examples, it is always able to find the exact solution with a reasonable amount of computational efforts.

- The semi-analytical method is capable of producing highly precise solutions comparable to that of the exact solutions. It outperforms the heuristic method in terms of computation 
time by roughly an order of magnitude.

- Ignoring user heterogeneity may severely mislead the analysis and design of step toll schemes in the bottleneck model.

A logical next step to this work is developing methods for solving the optimal step toll design problem under general heterogeneity, which is an ongoing effort. The heuristic version of the exact method proposed in this paper seems to offer a new and promising approach to the toll design problem. Other possible extensions include, but are not limited to: other dimensions of heterogeneity (such as desired arrival time), simultaneous departure time and route choices. As a final note, accommodating a discrete set of desired arrival times is relatively easy, since each desired arrival time would just divide the arrival window into more sub-windows, each corresponding to a unique arrival time and pricing structure. Consequently, such an extension would not change the analytical derivation of isocost curves as well as the nature of the formulation.

\section{Acknowledgement}

The work was partially supported by National Science Foundation under the award number CMMI-1256021, and by Singapore Ministry of Education Academic Research Fund Tier 1. We would also like to thank two anonymous reviewers and the Associate Editor, Professor Robin Lindsey, for their detailed and constructive comments on an earlier draft. The authors are responsible for the remaining errors.

\section{References}

Arnott, R., de Palma, A., Lindsey, R., 1988. Schedule delay and departure time decisions with heterogeneous commuters. Transportation Research Record 1197, 56-57.

Arnott, R., de Palma, A., Lindsey, R., 1990. Economics of a bottleneck. Journal of urban economics 27 (1), 111-130.

Arnott, R., de Palma, A., Lindsey, R., 1992. Route choice with heterogeneous drivers and groupspecific congestion costs. Regional Science and Urban Economics 22 (1), 71-102.

Arnott, R., de Palma, A., Lindsey, R., 1993. A structural model of peak-period congestion: A traffic bottleneck with elastic demand. The American Economic Review 83 (1), 161-179.

Arnott, R., de Palma, A., Lindsey, R., 1994. The welfare effects of congestion tolls with heterogeneous commuters. Journal of Transport Economics and Policy 28 (2), 139-161.

Arnott, R., Kraus, M., 1995. Financing capacity in the bottleneck model. Journal of Urban Economics 38 (3), 272-290.

Chu, X., 1999. Alternative congestion pricing schedules. Regional Science and Urban Economics 29 (6), 697-722. 
Cohen, Y., 1987. Commuter welfare under peak-period congestion tolls: Who gains and who loses? International Journal of Transport Economics= Rivista Internazionale de Economia dei Trasporti 14 (3), 238-266.

Daganzo, C. F., 1985. The uniqueness of a time-dependent equilibrium distribution of arrivals at a single bottleneck. Transportation science 19 (1), 29-37.

Hall, J., 2013. Pareto improvements from lexus lanes: the case for pricing a portion of the lanes on congested highways. In: Kuhmo NECTAR Conference.

Hendrickson, C., Kocur, G., 1981. Schedule delay and departure time decisions in a deterministic model. Transportation Science 15 (1), 62-77.

Higgins, T. J., 1994. Congestion pricing: Implementation considerations. Transportation Quarterly 48 (3), 287-298.

Laih, C.-H., 1994. Queueing at a bottleneck with single-and multi-step tolls. Transportation Research Part A 28 (3), 197-208.

Laih, C.-H., 2004. Effects of the optimal step toll scheme on equilibrium commuter behaviour. Applied Economics 36 (1), 59-81.

Lindsey, R., van den Berg, V. A., Verhoef, E. T., 2012. Step tolling with bottleneck queuing congestion. Journal of Urban Economics 72 (1), 46-59.

Lindsey, R., 2004. Existence, uniqueness, and trip cost function properties of user equilibrium in the bottleneck model with multiple user classes. Transportation science 38 (3), 293-314.

Liu, Y., 2013. Enabling congestion pricing: analysis and design of implementation strategies. Ph.D. thesis, Northwestern University.

Liu, Y., Nie, Y., 2011. Morning commute problem considering route choice, user heterogeneity and alternative system optima. Transportation Research Part B 45 (4), 619-642.

Liu, Y., Nie, Y., Hall, J., 2015. A semi-analytical approach for solving the bottleneck model with general user heterogeneity. Transportation research Part B 71, 56-70.

Newell, G. F., 1987. The morning commute for nonidentical travelers. Transportation Science $21(2), 74-88$.

Nie, Y., 2013. A new tradable credit scheme for the morning commute problem. Networks and Spatial Economics, Available at http:/ /dx.doi.org/10.1007/s11067-013-9192-8.

Small, K., 1982. The scheduling of comsumer activities: work trips. The American Economic Review 72 (3), 467-479.

Small, K., Winston, C., Yan, J., 2005. Uncovering the distribution of motorists' preferences for travel time and reliability. Econometrica 73 (4), 1367-1382. 
Smith, M. J., 1984. The existence of a time-dependent equilibrium distribution of arrivals at a single bottleneck. Transportation science 18 (4), 385-394.

van den Berg, V. A., 2014. Coarse tolling with heterogeneous preferences. Transportation Research Part B 64, 1-23.

van den Berg, V. A., Verhoef, E. T., 2011a. Congestion tolling in the bottleneck model with heterogeneous values of time. Transportation Research Part B 45 (1), 60-78.

van den Berg, V. A., Verhoef, E. T., 2011b. Winning or losing from dynamic bottleneck congestion pricing?: The distributional effects of road pricing with heterogeneity in values of time and schedule delay. Journal of Public Economics 95 (7-8), 983-992.

Vickrey, W., 1973. Pricing, metering, and efficiently using urban transportation facilities. Highway Research Record 476 (476), 36-48.

Vickrey, W., 1969. Congestion theory and transport investment. The American Economic Review 59 (2), 251-260.

Wardrop, J., 1952. Some theoretical aspects of road traffic research. Proceedings of the Institution of Civil Engineers, Part 2, 325-378.

Xiao, F. E., Qian, Z. S., Zhang, H. M., 2011. The morning commute problem with coarse toll and nonidentical commuters. Networks and Spatial Economics 11 (2), 343-369.

Yang, H., Huang, H.-J., 1997. Analysis of the time-varying pricing of a bottleneck with elastic demand using optimal control theory. Transportation Research Part B 31 (6), 425-440. 


\section{A Notation}

Table 4: Description of notations used in the paper

\begin{tabular}{|c|c|c|c|}
\hline Variable & Description & Unit & Range \\
\hline \multicolumn{4}{|c|}{ Bottleneck } \\
\hline $\begin{array}{l}s \\
T_{0} \\
T\end{array}$ & $\begin{array}{l}\text { capacity of the bottleneck } \\
\text { free flow travel time } \\
\text { waiting time at the bottleneck }\end{array}$ & $\begin{array}{r}\text { veh./ time } \\
\text { time } \\
\text { time }\end{array}$ & 0 \\
\hline \multicolumn{4}{|c|}{ Commuter } \\
\hline $\begin{array}{l}n \\
t^{*} \\
N_{i} \\
N=\sum_{i=1}^{n} N_{i} \\
\alpha_{i} \\
\beta_{i} \\
\gamma_{i}\end{array}$ & $\begin{array}{l}\text { number of user classes } \\
\text { desired arrival time } \\
\text { demand for class } i \\
\text { total demand } \\
\text { unit cost of travel time of class } i \\
\text { unit cost early arrival of class } i \\
\text { unit cost of late arrival of class } i\end{array}$ & $\begin{array}{r}- \\
\text { vehicle } \\
\text { vehicle } \\
\text { \$/unit time } \\
\text { \$/unit time } \\
\text { \$/unit time }\end{array}$ & $\begin{aligned} & 0 \\
i & =1,2, \ldots, n \\
i & =1,2, \ldots, n \\
i & =1,2, \ldots, n \\
i & =1,2, \ldots, n\end{aligned}$ \\
\hline \multicolumn{4}{|c|}{ Step toll } \\
\hline $\begin{array}{l}A \\
m^{A} \\
A_{j} \\
p_{j}^{A} \\
\pi_{j}^{A}\end{array}$ & $\begin{array}{l}\text { Arrival periods } \\
\text { number of toll steps in arrival period } A \\
j \text { th arrival window in arrival period } A \\
\text { Time distance between the far end of the ar- } \\
\text { rival window } A_{j} \text { and } t^{*} \\
\text { toll in the arrival window } A_{j}\end{array}$ & $\begin{array}{l}- \\
- \\
-\end{array}$ & $\begin{array}{c}E \text { (early), } L \text { (late) } \\
A=E, L \\
j=1, \ldots, m^{A}, A=E, L \\
j=1, \ldots, m^{A}, A=E, L \\
j=0,1, \ldots, m^{A}, A=E, L\end{array}$ \\
\hline \multicolumn{4}{|c|}{ Other } \\
\hline$E(i)$ & $\begin{array}{l}\text { class ID of the class whose } \frac{\beta}{\alpha} \text { value ranks at } \\
\text { the } i^{\text {th }} \text { place in all classes } \\
\text { class ID of the class whose } \frac{\gamma}{\alpha} \text { value ranks at } \\
\text { the } i^{\text {th }} \text { place in all classes }\end{array}$ & - & $\begin{array}{l}i=1,2, \ldots, n \\
i=1,2, \ldots, n\end{array}$ \\
\hline$A(i)$ & Generalization of $E(i)$ and $L(i)$ & - & $i=1,2, \ldots, n, A=E, L$ \\
\hline$n_{D}^{A_{j}}$ & $\begin{array}{l}\text { number of classes present in the arrival win- } \\
\text { dow } A_{j}\end{array}$ & - & $j=1, \ldots, m^{A}, A=E, L$ \\
\hline$D^{A_{j}}(i)$ & $\begin{array}{l}\text { ratio rank ID of the class whose time inflexi- } \\
\text { bility ranks at the } i^{\text {th }} \text { place among all classes } \\
\text { present in arrival period } A_{j}\end{array}$ & - & $i=1,2, \ldots, n_{D}^{A_{j}}, j=1, \ldots, m^{A}, A=E, L$ \\
\hline$N_{i}^{A_{j}}$ & $\begin{array}{l}\text { number of commuters of class } i \text { in the arrival } \\
\text { window } A_{j}\end{array}$ & vehicle & $i=1,2, \ldots, n, j=0,1, \ldots, m^{A}, A=E, L$ \\
\hline & vector of all $N_{i}^{A_{j}}$ & vehicle & \\
\hline$C_{i}^{A_{j}}\left(c_{i}^{A_{j}}\right)$ & $\begin{array}{l}\text { actual commute cost of class } i \text { in the arrival } \\
\text { window } A_{j} \text {, measured in travel time (mone- } \\
\text { tary value) }\end{array}$ & time (\$) & $i=1,2, \ldots, n, j=0,1, \ldots, m^{A}, A=E, L$ \\
\hline$C(c)$ & $\begin{array}{l}\text { vector of all } C_{i}^{A_{j}} \text {, measured in travel time } \\
\text { (monetary value) }\end{array}$ & time $(\$)$ & \\
\hline$\mu_{i}$ & UE cost of class $i$, in travel time & time & $i=1,2, \ldots, n$ \\
\hline $\begin{array}{l}\mu \\
v_{j}^{A}\end{array}$ & $\begin{array}{l}\text { vector of UE cost } \\
\text { multiplier associated with the capacity con- } \\
\text { straint for the arrival window } A_{j}\end{array}$ & time & $j=1, \ldots, m^{A}, A=E, L$ \\
\hline$\rho_{i}^{A_{j}}$ & $\begin{array}{l}\text { class membership set realization (indicating } \\
\text { whether class } i \text { is present in } A_{j} \text { ) } \\
\text { vector of } \rho_{i}^{A_{j}}\end{array}$ & & $i=1,2, \ldots, n, j=0,1, \ldots, m^{A}, A=E, L$ \\
\hline
\end{tabular}




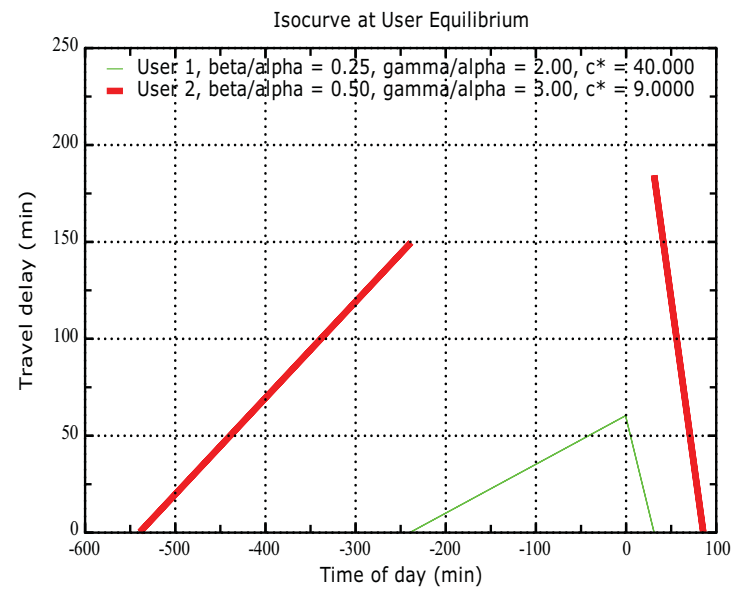

(a) Solution with Lowest Cost for Class 1

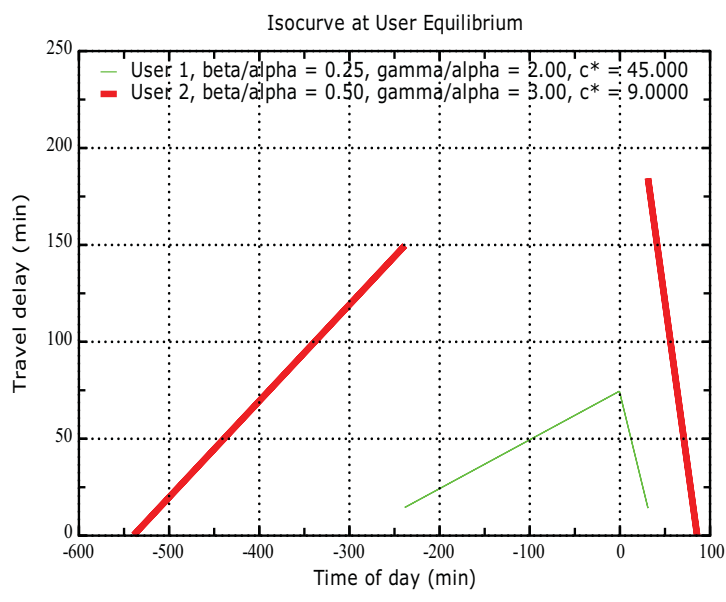

(b) Solution with Highest Cost for Class 1

Figure 6: Illustration of non-uniqueness in UE cost

\section{B Example of non-unique UE cost}

In this example, the bottleneck capacity $s=1000$ (veh/hour) and a single step toll of $\$ 20$ is applied for an arrival window $[-4,0.5]$, where $t^{*}=0$. The details of the classes and the UE cost solutions are given in Table 5 . The reader can verify that for class 1 , the UE cost $\mu_{1}$ range between 2 and 2.5 (hour). However, there is a unique flow pattern (hence a unique CMSR) corresponding to all those different UE costs. Two extreme solutions (corresponding to $\mu_{1}=2,2.5$ ) are shown in Figure 6. It is clear from the figure that the difference is derived from the multipliers associated with the arrival window, which can range between 0 to 0.5 (hour).

Table 5: Class information and UE cost

\begin{tabular}{|c|c|c|c|c|c|c|c|}
\hline Class No. & Demand & \multicolumn{3}{|c|}{ Values of Time } & \multicolumn{2}{|c|}{ Time Inflexibility } & UE Cost \\
\hline$i$ & $N_{i}$ & $\alpha_{i}(\$ /$ hour $)$ & $\beta_{i}(\$ /$ hour $)$ & $\gamma_{i}(\$ /$ hour $)$ & $\frac{\beta_{i}}{\alpha_{i}}$ & $\frac{\gamma_{i}}{\alpha_{i}}$ & $\mu_{i}^{*}$ (hour) \\
\hline 1 & 4500 & 20 & 5 & 40 & $1 / 4$ & 2 & {$[2,2.5]$} \\
\hline 2 & 5500 & 2 & 1 & 6 & $1 / 2$ & 3 & 4.5 \\
\hline
\end{tabular}




\section{Details of the two-class example}

\section{C.1 Semi-analytical method}

For this problem, we have eight flows (four for each user class) and two capacity multipliers (one for each tolled window) to balance. From Equation (8), we further write the costs as

$$
\begin{array}{ll}
C_{1}^{E_{0}}=\frac{1}{3000} N_{1}^{E_{0}}+\frac{1}{3000} N_{2}^{E_{0}}+\frac{4}{3}, & C_{2}^{E_{0}}=\frac{1}{3000} N_{1}^{E_{0}}+\frac{3}{4000} N_{2}^{E_{0}}+3, \\
C_{1}^{E_{1}}+v_{1}^{E}=\frac{1}{3000} N_{1}^{E_{1}}+\frac{1}{3000} N_{2}^{E_{1}}+\frac{5}{3}+v_{1}^{E}, & C_{2}^{E_{1}}+v_{1}^{E}=\frac{1}{3000} N_{1}^{E_{1}}+\frac{3}{4000} N_{2}^{E_{1}}+\frac{5}{2}+v_{1}^{E}, \\
C_{1}^{L_{1}}+v_{1}^{L}=\frac{5}{2000} N_{1}^{L_{1}}+\frac{5}{4000} N_{2}^{L_{1}}+\frac{5}{3}+v_{1}^{L}, & C_{2}^{L_{1}}+v_{1}^{L}=\frac{5}{4000} N_{1}^{L_{1}}+\frac{5}{4000} N_{2}^{L_{1}}+\frac{5}{2}+v_{1}^{L}, \\
C_{1}^{L_{0}}=\frac{5}{2000} N_{1}^{L_{0}}+\frac{5}{4000} N_{2}^{L_{0}}+\frac{5}{2}, & C_{2}^{L_{0}}=\frac{5}{4000} N_{1}^{L_{0}}+\frac{5}{4000} N_{2}^{L_{0}}+\frac{5}{4} .
\end{array}
$$

According to Equation (5b), the capacity constraints are

$$
\begin{aligned}
& N_{1}^{E_{1}}+N_{2}^{E_{1}} \leq 4000, \\
& N_{1}^{L_{1}}+N_{2}^{L_{1}} \leq 1000 .
\end{aligned}
$$

For the first outer iteration and its first inner iteration, $\mathbf{v}=\mathbf{0}$ and $\mathbf{N}=\mathbf{0}$. For Class 1:

$C_{1}^{E_{0}}=\frac{1}{3000} N_{1}^{E_{0}}+\frac{4}{3}, \quad C_{1}^{E_{1}}+v_{1}^{E}=\frac{1}{3000} N_{1}^{E_{1}}+\frac{5}{3}, \quad C_{1}^{L_{1}}+v_{1}^{L}=\frac{5}{2000} N_{1}^{L_{1}}+\frac{5}{3}, \quad C_{1}^{L_{0}}=\frac{5}{2000} N_{1}^{L_{0}}+\frac{5}{2} ;$

and

$$
N_{1}^{E_{0}}+N_{1}^{E_{1}}+N_{1}^{L_{1}}+N_{1}^{L_{0}}=5000 .
$$

Hence, by sorting the intercepts, we find that the flows should be assigned according to the following order: $E_{0}, E_{1}, L_{1}$, and $L_{0}$. The equilibrated flows are

$$
N_{1}^{E_{0}}=2875, \quad N_{1}^{E_{1}}=1875, \quad N_{1}^{L_{1}}=250, \quad N_{1}^{L_{0}}=0 .
$$

Fixing the flows of Class 1, we have now for class 2:

$C_{2}^{E_{0}}=\frac{3}{4000} N_{2}^{E_{0}}+\frac{95}{24}, \quad C_{2}^{E_{1}}+v_{1}^{E}=\frac{3}{4000} N_{2}^{E_{1}}+\frac{25}{8}, \quad C_{2}^{L_{1}}+v_{1}^{L}=\frac{5}{4000} N_{2}^{L_{1}}+\frac{45}{16}, \quad C_{2}^{L_{0}}=\frac{5}{4000} N_{2}^{L_{0}}+\frac{5}{4} ;$

and

$$
N_{2}^{E_{0}}+N_{2}^{E_{1}}+N_{2}^{L_{1}}+N_{2}^{L_{0}}=5000 .
$$

This time, the flows should be assigned in the order of $L_{0}, L_{1}, E_{1}$, and $E_{0}$. The equilibrated flows are

$$
N_{2}^{E_{0}}=\frac{18125}{72}, \quad N_{2}^{E_{1}}=\frac{98125}{72}, \quad N_{2}^{L_{1}}=\frac{25625}{24}, \quad N_{2}^{L_{0}}=\frac{55625}{24} .
$$


By now, the first inner iteration is completed. After repeating inner iterations 17 times, the algorithm converges to the unconstrained UE flow solution corresponding to $\mathbf{v}=0$ :

$$
\begin{array}{llll}
N_{1}^{E_{0}}=3758.62, & N_{1}^{E_{1}}=1241.38, & N_{1}^{L_{1}}=0, & N_{1}^{L_{0}}=0 ; \\
N_{2}^{E_{0}}=0, & N_{2}^{E_{1}}=1517.24, & N_{2}^{L_{1}}=1241.38, & N_{2}^{L_{0}}=2241.38 .
\end{array}
$$

Now, with this unconstrained UE flow solution, the algorithm starts to modify the capacity multipliers in the outer loop. First, by checking the capacity constraints, we have

$$
\begin{aligned}
& N_{1}^{E_{1}}+N_{2}^{E_{1}}=2758.62 \leq 4000 \\
& N_{1}^{L_{1}}+N_{2}^{L_{1}}=1241.38>1000 .
\end{aligned}
$$

Hence, the multiplier $v_{1}^{E}$ should be decreased while $v_{1}^{L}$ should be increased. Since $v_{1}^{E}$ is already zero, no changes is needed. For the arrival window $L_{1}, \Delta=241.38$, and Class 2 with a late time inflexibility of $\frac{5}{4}$ should be penalized. Hence, the increase amount in $v_{1}^{L}$ should be $\left(\frac{241.38}{1000} \times \frac{5}{4}\right)=$ 0.30173. Therefore, after the first outer iteration, the values of multipliers should be

$$
v_{1}^{E}=0, \quad v_{1}^{L}=0.30173 .
$$

The algorithm then resumes the inner loop with the new $\mathbf{v}$, until both the multipliers and flows converge. The reader can verify that the converged flow solution is

$$
\begin{array}{llll}
N_{1}^{E_{0}}=3840.91, & N_{1}^{E_{1}}=1159.09, & N_{1}^{L_{1}}=0, & N_{1}^{L_{0}}=0 \\
N_{2}^{E_{0}}=0, & N_{2}^{E_{1}}=1681.82, & N_{2}^{L_{1}}=1000.00, & N_{2}^{L_{0}}=2318.18 .
\end{array}
$$

\section{C.2 Exact method}

First, it is easy to verify that even in such a simple example, the number of total CMSRs is 784, which makes it impossible to demonstrate the whole process of exact method by enumeration. Since for exact method, the processes of solving linear equation systems are the same for both enumeration algorithm and heuristic algorithm, we will only illustrate how heuristic algorithm solves the step-tolled UE problem with two classes.

The isocost curves for user classes can be written as

$$
T_{0}(t)=0 ; T_{1}(t)=\left\{\begin{array}{cc}
\frac{1}{3} t+\mu_{1}, & t<-4 \\
\frac{1}{3} t+\mu_{1}-\frac{5}{3}, & -4 \leq t<0 \\
-\frac{5}{2} t+\mu_{1}-\frac{5}{3}, & 0 \leq t<1 \\
-\frac{5}{2} t+\mu_{1}, & t \geq 1
\end{array} \quad ; T_{2}(t)=\left\{\begin{array}{cc}
\frac{3}{4} t+\mu_{2}, & t<-4 \\
\frac{3}{4} t+\mu_{2}-\frac{5}{2}, & -4 \leq t<0 \\
-\frac{5}{4} t+\mu_{2}-\frac{5}{2}, & 0 \leq t<1 \\
-\frac{5}{4} t+\mu_{2}, & t \geq 1
\end{array} .\right.\right.
$$

The starting CMSR assumes all classes are present, which is

$$
\rho^{0}=\left\{\rho^{E_{0}}, \rho^{E_{1}}, \rho^{L_{1}}, \rho^{L_{0}}\right\}=\{(0,1,2),(0,1,2),(0,1,2),(0,1,2)\} .
$$

With this $\rho^{0}$ given, from Equation (17), we can get the horizontal coordinates for all presenting 
classes

$$
\begin{array}{rlrlrl}
x_{0}^{E_{0}} & =-\infty, & x_{1}^{E_{0}} & =-3 \mu_{1}, & x_{2}^{E_{0}}=\frac{12}{5} \mu_{1}-\frac{12}{5} \mu_{2}, & x_{3}^{E_{0}}=-4, \\
x_{0}^{E_{1}}=-4, & x_{1}^{E_{1}}=-3 \mu_{1}+5, & x_{2}^{E_{1}}=\frac{12}{5} \mu_{1}-\frac{12}{5} \mu_{2}+2, & x_{3}^{E_{1}}=0, \\
x_{0}^{L_{1}}=1, & x_{1}^{L_{1}}=\frac{4}{5} \mu_{1}-\frac{4}{5} \mu_{2}+\frac{2}{3}, & x_{2}^{L_{1}}=\frac{4}{5} \mu_{2}-2, & x_{3}^{L_{1}}=0, \\
x_{0}^{L_{0}}=+\infty, & x_{1}^{L_{0}}=\frac{4}{5} \mu_{1}-\frac{4}{5} \mu_{2}, & x_{2}^{L_{0}}=\frac{4}{5} \mu_{2}, & x_{3}^{L_{0}}=1 .
\end{array}
$$

Hence, from Equation (18), the flows of real classes in each window are

$$
\begin{aligned}
\frac{N_{1}^{E_{0}}}{1000} & =\frac{27}{5} \mu_{1}-\frac{12}{5} \mu_{2}, & \frac{N_{2}^{E_{0}}}{1000} & =-\frac{12}{5} \mu_{1}+\frac{12}{5} \mu_{2}-4, \\
\frac{N_{1}^{E_{1}}}{1000} & =\frac{27}{5} \mu_{1}-\frac{12}{5} \mu_{2}-3, & \frac{N_{2}^{E_{1}}}{1000} & =-\frac{12}{5} \mu_{1}+\frac{12}{5} \mu_{2}-2, \\
\frac{N_{1}^{L_{1}}}{1000} & =\frac{4}{5} \mu_{1}-\frac{4}{5} \mu_{2}+\frac{2}{3}, & \frac{N_{2}^{L_{1}}}{1000} & =-\frac{4}{5} \mu_{1}+\frac{8}{5} \mu_{2}-\frac{8}{3}, \\
\frac{N_{1}^{L_{0}}}{1000} & =\frac{4}{5} \mu_{1}-\frac{4}{5} \mu_{2}-1, & \frac{N_{2}^{L_{0}}}{1000} & =-\frac{4}{5} \mu_{1}+\frac{8}{5} \mu_{2} .
\end{aligned}
$$

By Equation (19), we can set up the 2-dimensional linear equation system of $\mu$ as

$$
\begin{aligned}
& \frac{62}{5} \mu_{1}-\frac{32}{5} \mu_{2}-\frac{10}{3}=5, \\
& -\frac{32}{5} \mu_{1}+8 \mu_{2}-\frac{26}{3}=5 .
\end{aligned}
$$

Hence, for $\rho^{0}$, the general costs in travel delay should be $\left(\mu_{1}, \mu_{2}\right)=(2.64652,3.82555)$.

We now examine this solution against constraints (20-23), i.e.,

$$
\begin{array}{llll}
\text { For } E_{0}: & -3 \mu_{1}+\infty \geq 0, & \frac{27}{5} \mu_{1}-\frac{12}{5} \mu_{2} \geq 0, & -\frac{12}{5} \mu_{1}+\frac{12}{5} \mu_{2}-4 \geq 0 ; \\
\text { For } E_{1}: & -3 \mu_{1}+9 \geq 0, & \frac{27}{5} \mu_{1}-\frac{12}{5} \mu_{2}-3 \geq 0, & -\frac{12}{5} \mu_{1}+\frac{12}{5} \mu_{2}-2 \geq 0 ; \\
\text { For } L_{1}: & -\frac{4}{5} \mu_{2}+3 \geq 0, & \frac{4}{5} \mu_{1}-\frac{4}{5} \mu_{2}+\frac{2}{3} \geq 0, & -\frac{4}{5} \mu_{1}+\frac{8}{5} \mu_{2}-\frac{8}{3} \geq 0 ; \\
\text { For } L_{0}: & -\frac{4}{5} \mu_{2}+\infty \geq 0, & \frac{4}{5} \mu_{1}-\frac{4}{5} \mu_{2}-1 \geq 0, & -\frac{4}{5} \mu_{1}+\frac{8}{5} \mu_{2} \geq 0 .
\end{array}
$$

Only for window $E_{1}$, all three constraints are satisfied. The constraints that are violated are: the third one for $E_{0}$, the first one and second one for $L_{1}$, and the second one for $L_{0}$. The most severely violated constraints for each window are: the third one for $E_{0}$ (associated with Class 2), the second one for $L_{1}$ (associated with Class 1), and the second one for $L_{0}$ (associated with Class 1). Hence, the new CMSR to go in the heuristic algorithm should be

$$
\rho^{1}=\left\{\rho^{E_{0}}, \rho^{E_{1}}, \rho^{L_{1}}, \rho^{L_{0}}\right\}=\{(0,1),(0,1,2),(0,2),(0,2)\} .
$$

The new $\rho$ gives rise to a solution of $\left(\mu_{1}, \mu_{2}\right)=(2.58621,4.05172)$. For this solution, the constraints are all satisfied for windows $E_{0}, E_{1}$ and $L_{0}$. For window $L_{1}$, the most severely violated 
constraint is associated with Class 0 . Hence, the new CMSR becomes

$$
\rho^{2}=\left\{\rho^{E_{0}}, \rho^{E_{1}}, \rho^{L_{1}}, \rho^{L_{0}}\right\}=\{(0,1),(0,1,2),(2),(0,2)\} .
$$

The solution corresponding to $\rho^{2}$ is $\left(\mu_{1}, \mu_{2}\right)=(2.61364,4.14773)$. The reader can verify that in this case all constraints are satisfied. Consequently, the heuristic algorithm finds the UE solution after only three iterations (the exact method would need to iterate 784 times in the worst case). 